\title{
Functoriality of the canonical fractional Galois ideal
}

\author{
Paul Buckingham Victor Snaith
}

\begin{abstract}
The fractional Galois ideal of [Victor P. Snaith, Stark's conjecture and new Stickelberger phenomena, Canad. J. Math. 58 (2) (2006) 419-448] is a conjectural improvement on the higher Stickelberger ideals defined at negative integers, and is expected to provide non-trivial annihilators for higher $K$-groups of rings of integers of number fields. In this article, we extend the definition of the fractional Galois ideal to arbitrary (possibly infinite and non-abelian) Galois extensions of number fields under the assumption of Stark's conjectures, and prove naturality properties under canonical changes of extension. We discuss applications of this to the construction of ideals in non-commutative Iwasawa algebras.
\end{abstract}

\section{Introduction}

Let $E / F$ be a Galois extension of number fields with Galois group $G$. In seeking annihilators in $\mathbb{Z}[G]$ of the $K$-groups $K_{2 n}\left(\mathcal{O}_{E, S}\right)(S$ a finite set of places of $E$ containing the infinite ones), Stickelberger elements have long been a source of interest. This began with the classical Stickelberger theorem, showing that for abelian extensions $E / \mathbb{Q}$, annihilators of $\operatorname{Tors}\left(K_{0}\left(\mathcal{O}_{E, S}\right)\right)$ can be constructed from Stickelberger elements. Coates and Sinnott later conjectured in 12] that the analogous phenomenon would occur for higher $K$-groups. However, defined in terms of values of $L$-functions at negative integers, these elements do not provide all the annihilators, because of the prevalent vanishing of the $L$-function values.

This difficulty is hoped to be overcome by considering the "fractional Galois ideal" introduced by the second author in [33, 34 and defined in terms of leading coefficients of $L$-functions at negative integers under the assumption of the higher Stark conjectures. A version more suitable for the case of $\operatorname{Tors}\left(K_{0}\left(\mathcal{O}_{E, S}\right)\right)=\mathrm{Cl}\left(\mathcal{O}_{E, S}\right)$ was defined in [5] by the first author. Evidence that the fractional Galois ideal annihilates the appropriate $K$-groups (resp. class-groups) can be found in [34] (resp. [5]). In the first case, it is étale cohomology that is annihilated, but this is expected to give $K$-theory by the Lichtenbaum-Quillen conjecture (see [34, Section 1] for details).

With a view to relating the fractional Galois ideal to characteristic ideals in Iwasawa theory, we would like to describe how it behaves in towers of number 
fields. That it exhibits naturality in certain changes of extension was observed in particular cases in [5, and part of the aim of this paper is to explain these phenomena generally. Passage to subextensions corresponding to quotients of Galois groups will be of particular interest in the situation of non-abelian extensions, because of the relatively recent emergence of non-commutative Iwasawa theory in, for example, [11, 15. Consequently, the aims of this paper are

(i) to prove formal properties of the fractional Galois ideal with respect to changes of extension, in the commutative setting first ( 3.3 to $\$ 3.6$ )

(ii) to extend the definition of the fractional Galois ideal to nonabelian Galois extensions (\$5), having previously defined it only for abelian extensions

(iii) to show that it behaves well under passing to subextensions in the non-commutative setting also (Proposition 5.3)

(iv) to show that in order for the non-commutative fractional Galois ideals to annihilate the appropriate étale cohomology groups, it is sufficient that the commutative ones do (\$77).

We will also provide an explicit example (in the commutative case) in \$6.2.1 illustrating how a limit of fractional Galois ideals gives the Fitting ideal for an inverse limit $\mathrm{Cl}_{\infty}$ of $\ell$-parts of class-groups. This should make clear the importance of taking leading coefficients of $L$-functions rather than just values, since it will be the part of the fractional Galois ideal corresponding to $L$-functions with first-order vanishing at 0 which provides the Fitting ideal for the plus-part of $\mathrm{Cl}_{\infty}$.

In 88 , we will conclude with a discussion of how the constructions of this paper fit into non-commutative Iwasawa theory. In particular, under some assumptions which, compared with the many conjectures permeating this area, are relatively weak, we will be able to give a partial answer to a question of Ardakov-Brown in [1] on constructing ideals in Iwasawa algebras.

\subsection{Error in Proposition 3.6}

Since the acceptance of the paper, the authors were made aware of a problem in Proposition 3.6. It has to do with the fact that the induction map on representations is an additive homomorphism of representation rings, while the functoriality of $L$-functions refers to multiplication. Contrary to our expectations at the time, we have not been able to resolve this issue. We thank Andreas Nickel for bringing this problem to our attention.

\section{Notation and the Stark conjectures}

In what follows, by a Galois representation of a number field $F$ we shall mean a continuous, finite-dimensional complex representation of the absolute Galois group of $F$, which amounts to saying that the representation factors through the Galois group $\operatorname{Gal}(E / F)$ of a finite Galois extension $E / F$. We begin with 
the Stark conjecture (at $s=0$ ) and its generalizations to $s=-1,-2,-3, \ldots$ which were introduced in [16] and 34] independently.

Let $\Sigma(E)$ denote the set of embeddings of $E$ into the complex numbers. For $r=0,-1,-2,-3, \ldots$ set

$$
Y_{r}(E)=\prod_{\Sigma(E)}(2 \pi i)^{-r} \mathbb{Z}=\operatorname{Map}\left(\Sigma(E),(2 \pi i)^{-r} \mathbb{Z}\right)
$$

endowed with the $G(\mathbb{C} / \mathbb{R})$-action diagonally on $\Sigma(E)$ and on $(2 \pi i)^{-r}$. If $c_{0}$ denotes complex conjugation, the action of $c_{0}$ and $G$ commute so that the fixed points of $Y_{r}(E)$ under $c_{0}$, denoted by $Y_{r}(E)^{+}$, form a $G$-module. It is easy to see that the rank of $Y_{r}(E)^{+}$is given by

$$
\operatorname{rk}_{\mathbb{Z}}\left(Y_{r}(E)^{+}\right)= \begin{cases}r_{2} & \text { if } r \text { is odd } \\ r_{1}+r_{2} & \text { if } r \geq 0 \text { is even. }\end{cases}
$$

where $|\Sigma(E)|=r_{1}+2 r_{2}$ and $r_{1}$ is the number of real embeddings of $E$.

\subsection{Stark regulators}

We begin with a slight modification of the original Stark regulator [36]. Now let $G$ denote the Galois group of an extension of number fields $E / F$. We extend the Dirichlet regulator homomorphism to the Laurent polynomials with coefficients in $\mathcal{O}_{E}$ to give an $\mathbb{R}[G]$-module isomorphism of the form

$$
R_{E}^{0}: K_{1}\left(\mathcal{O}_{E}\left\langle t^{ \pm 1}\right\rangle\right) \otimes \mathbb{R}=\mathcal{O}_{E}\left\langle t^{ \pm 1}\right\rangle^{\times} \otimes \mathbb{R} \cong Y_{0}(E)^{+} \otimes \mathbb{R} \cong \mathbb{R}^{r_{1}+r_{2}}
$$

by the formulae, for $u \in \mathcal{O}_{E}^{\times}$,

$$
R_{E}^{0}(u)=\sum_{\sigma \in \Sigma(E)} \log (|\sigma(u)|) \cdot \sigma
$$

and

$$
R_{E}^{0}(t)=\sum_{\sigma \in \Sigma(E)} \sigma
$$

The existence of this isomorphism implies (see [30, Section 12.1] and [36, p.26]) that there exists at least one $\mathbb{Q}[G]$-module isomorphism of the form

$$
f_{E}^{0}: \mathcal{O}_{E}\left\langle t^{ \pm 1}\right\rangle^{\times} \otimes \mathbb{Q} \stackrel{\cong}{\rightrightarrows} Y_{0}(E)^{+} \otimes \mathbb{Q} .
$$

For any choice of $f_{E}^{0}$ Stark forms the composition

$$
R_{E}^{0} \cdot\left(f_{E}^{0}\right)^{-1}: Y_{0}(E)^{+} \otimes \mathbb{C} \stackrel{\cong}{\rightarrow} Y_{0}(E)^{+} \otimes \mathbb{C}
$$

which is an isomorphism of complex representations of $G$. Let $V$ be a finitedimensional complex representation of $G$ whose contragredient is denoted by 
$V^{\vee}$. The Stark regulator is defined to be the exponential homomorphism $V \mapsto$ $R\left(V, f_{E}^{0}\right)$, from representations to non-zero complex numbers, given by

$$
R\left(V, f_{E}^{0}\right)=\operatorname{det}\left(\left(R_{E}^{0} \cdot\left(f_{E}^{0}\right)^{-1}\right)_{*} \in \operatorname{Aut}_{\mathbf{C}}\left(\operatorname{Hom}_{G}\left(V^{\vee}, Y_{0}(E)^{+} \otimes \mathbb{C}\right)\right)\right)
$$

where $\left(R_{E}^{0} \cdot\left(f_{E}^{0}\right)^{-1}\right)_{*}$ is composition with $R_{E}^{0} \cdot\left(f_{E}^{0}\right)^{-1}$.

For $r=-1,-2,-3, \ldots$ there is an isomorphism of the form [26]

$$
K_{1-2 r}\left(\mathcal{O}_{E}\left\langle t^{ \pm 1}\right\rangle\right) \otimes \mathbb{Q} \cong K_{1-2 r}\left(\mathcal{O}_{E}\right) \otimes \mathbb{Q}
$$

because $K_{-2 r}\left(\mathcal{O}_{E}\right)$ is finite. Therefore the Borel regulator homomorphism defines an $\mathbb{R}[G]$-module isomorphism of the form

$$
R_{E}^{r}: K_{1-2 r}\left(\mathcal{O}_{E}\left\langle t^{ \pm 1}\right\rangle\right) \otimes \mathbb{R}=K_{1-2 r}\left(\mathcal{O}_{E}\right) \otimes \mathbb{R} \stackrel{\cong}{\rightrightarrows} Y_{r}(E)^{+} \otimes \mathbb{R} .
$$

Choose a $\mathbb{Q}[G]$-module isomorphism of the form

$$
f_{E}^{r}: K_{1-2 r}\left(\mathcal{O}_{E}\left\langle t^{ \pm 1}\right\rangle\right) \otimes \mathbb{Q} \stackrel{\cong}{\rightrightarrows} Y_{r}(E)^{+} \otimes \mathbb{Q}
$$

and form the analogous Stark regulator, $\left(V \mapsto R\left(V, f_{E}^{r}\right)\right)$, from representations to non-zero complex numbers given by

$$
R\left(V, f_{E}^{r}\right)=\operatorname{det}\left(\left(R_{E}^{r} \cdot\left(f_{E}^{r}\right)^{-1}\right)_{*} \in \operatorname{Aut}_{\mathbf{C}}\left(\operatorname{Hom}_{G}\left(V^{\vee}, Y_{r}(E)^{+} \otimes \mathbb{C}\right)\right)\right) .
$$

\subsection{Stark's conjectures}

Let $R(G)$ denote the complex representation ring of the finite group $G$; that is, $R(G)=K_{0}(\mathbb{C}[G])$. Since $V$ determines a Galois representation of $F$, we have a non-zero complex number $L_{F}^{*}(r, V)$ given by the leading coefficient of the Taylor series at $s=r$ of the Artin $L$-function associated to $V$ ([23], [36, p.23]).

We may modify $R\left(V, f_{E}^{r}\right)$ to give another exponential homomorphism

$$
\mathcal{R}_{f_{E}^{r}} \in \operatorname{Hom}\left(R(G), \mathbb{C}^{\times}\right)
$$

defined by

$$
\mathcal{R}_{f_{E}^{r}}(V)=\frac{R\left(V, f_{E}^{r}\right)}{L_{F}^{*}(r, V)} .
$$

Let $\overline{\mathbb{Q}}$ denote the algebraic closure of the rationals in the complex numbers and let $\Omega_{\mathbb{Q}}$ denote the absolute Galois group of the rationals, which acts continuously on $R(G)$ and $\overline{\mathbb{Q}}^{\times}$. The Stark conjecture asserts that for each $r=$ $0,-1,-2,-3, \ldots$

$$
\mathcal{R}_{f_{E}^{r}} \in \operatorname{Hom}_{\Omega_{\mathbb{Q}}}\left(R(G), \overline{\mathbb{Q}}^{\times}\right) \subseteq \operatorname{Hom}\left(R(G), \mathbb{C}^{\times}\right) .
$$

In other words, $\mathcal{R}_{f_{E}^{r}}(V)$ is an algebraic number for each $V$ and for all $z \in \Omega_{\mathbb{Q}}$ we have $z\left(\mathcal{R}_{f_{E}^{r}}(V)\right)=\mathcal{R}_{f_{E}^{r}}(z(V))$. Since any two choices of $f_{E}^{r}$ differ by multiplication by a $\mathbb{Q}[G]$-automorphism, the truth of the conjecture is independent of the choice of $f_{E}^{r}([36$ pp.28-30). 
When $s=0$ the conjecture which we have just formulated apparently differs from the classical Stark conjecture of [36, therefore we shall pause to show that the two conjectures are equivalent. For the classical Stark conjecture one replaces $Y_{0}(E)^{+}$by $X_{0}(E)^{+}$where $X_{0}(E)$ is the kernel of the augmentation homomorphism $Y_{0}(E) \rightarrow \mathbb{Z}$, which adds together all the coordinates. The Dirichlet regulator gives an $\mathbb{R}[G]$-module isomorphism

$$
\tilde{R}_{E}^{0}: \mathcal{O}_{E}^{\times} \otimes \mathbb{R} \cong X_{0}(E)^{+} \otimes \mathbb{R}
$$

and choosing a $\mathbb{Q}[G]$-module isomorphism

$$
\tilde{f}_{E}^{0}: \mathcal{O}_{E}^{\times} \otimes \mathbb{Q} \stackrel{\cong}{\rightarrow} X_{0}(E)^{+} \otimes \mathbb{Q}
$$

we may form

$$
\tilde{R}_{E}^{0} \cdot\left(\tilde{f}_{E}^{0}\right)^{-1}: X_{0}(E)^{+} \otimes \mathbb{C} \stackrel{\cong}{\rightrightarrows} X_{0}(E)^{+} \otimes \mathbb{C} .
$$

Taking its Stark determinant we obtain $\tilde{R}\left(V, \tilde{f}_{E}^{0}\right)$ and finally

$$
\tilde{\mathcal{R}}_{\tilde{f}_{E}^{0}}(V)=\frac{\tilde{R}\left(V, \tilde{f}_{E}^{0}\right)}{L_{F}^{*}(0, V)} .
$$

Proposition 2.1 In 2.2

$$
\mathcal{R}_{f_{E}^{0}} \in \operatorname{Hom}_{\Omega_{\mathbb{Q}}}\left(R(G), \overline{\mathbb{Q}}^{\times}\right) \subseteq \operatorname{Hom}\left(R(G), \mathbb{C}^{\times}\right)
$$

if and only if

$$
\tilde{\mathcal{R}}_{\tilde{f}_{E}^{0}} \in \operatorname{Hom}_{\Omega_{\mathbb{Q}}}\left(R(G), \overline{\mathbb{Q}}^{\times}\right) \subseteq \operatorname{Hom}\left(R(G), \mathbb{C}^{\times}\right)
$$

independently of the choice of $f_{E}^{0}$ or $\tilde{f}_{E}^{0}$.

Proof. Given any $\mathbb{Q}[G]$-isomorphism $\tilde{f}_{E}^{0}$ we may fill in the following commutative diagram by $\mathbb{Q}[G]$-isomorphisms $f_{E}^{0}$ and $\bar{f}_{E}^{0}$. Conversely, given any $\mathbb{Q}[G]$ isomorphisms $f_{E}^{0}$ and $\bar{f}_{E}^{0}$ we may fill in the diagram with a $\mathbb{Q}[G]$-isomorphism $\tilde{f}_{E}^{0}$.

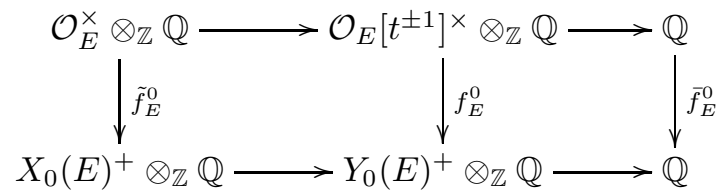

Similarly there is a commutative diagram in which the vertical arrows are reversed, $\mathbb{Q}$ is replaced by $\mathbb{R}$ and $\tilde{f}_{E}, f_{E}$ and $\bar{f}_{E}$ by $\tilde{R}_{E}^{0}, R_{E}^{0}$ and $\bar{R}_{E}^{0}$, respectively. Furthermore $\bar{R}_{E}^{0}$ is multiplication by a rational number. The result now follows from the multiplicativity of the determinant in short exact sequences.

We shall be particularly interested in the case when $G$ is abelian, in which case the following observation is important. Let $\widehat{G}=\operatorname{Hom}\left(G, \overline{\mathbb{Q}}^{\times}\right)$denote the 
set of characters on $G$ and let $\mathbb{Q}(\chi)$ denote the field generated by the character values of a representation $\chi$. We may identify $\operatorname{Hom}_{\Omega_{\mathbb{Q}}}(R(G), \overline{\mathbb{Q}})$ with the ring $\operatorname{Map}_{\Omega_{\mathbb{Q}}}(\widehat{G}, \overline{\mathbb{Q}})$.

Proposition 2.2 Let $G$ be a finite abelian group. Then there exists an isomorphism of rings

$$
\lambda_{G}: \operatorname{Map}_{\Omega_{\mathbb{Q}}}(\widehat{G}, \overline{\mathbb{Q}})=\operatorname{Hom}_{\Omega_{\mathbb{Q}}}(R(G), \overline{\mathbb{Q}}) \stackrel{\cong}{\rightarrow}[G]
$$

given by

$$
\lambda_{G}(h)=\sum_{\chi \in \widehat{G}} h(\chi) e_{\chi}
$$

where

$$
e_{\chi}=|G|^{-1} \sum_{g \in G} \chi(g) g^{-1} \in \mathbb{Q}(\chi)[G] .
$$

In particular there is an isomorphism of unit groups

$$
\lambda_{G}: \operatorname{Hom}_{\Omega_{\mathbb{Q}}}\left(R(G), \overline{\mathbb{Q}}^{\times}\right) \stackrel{\cong}{\rightarrow} \mathbb{Q}[G]^{\times} .
$$

Proof. There is a well-known isomorphism of rings (22] p.648)

$$
\psi: \overline{\mathbb{Q}}[G] \rightarrow \prod_{\chi \in \widehat{G}} \overline{\mathbb{Q}}=\operatorname{Map}(\widehat{G}, \overline{\mathbb{Q}})
$$

given by $\psi\left(\sum_{g \in G} \lambda_{g} g\right)(\chi)=\sum_{g \in G} \lambda_{g} \chi(g)$. If $\Omega_{\mathbb{Q}}$ acts on $\overline{\mathbb{Q}}$ and $\widehat{G}$ in the canonical manner, then $\psi$ is Galois equivariant and induces an isomorphism of $\Omega_{\mathbb{Q}}$-fixed points of the form

$$
\mathbb{Q}[G]=(\overline{\mathbb{Q}}[G])^{\Omega_{\mathbb{Q}}} \cong \operatorname{Map}_{\Omega_{\mathbb{Q}}}(\widehat{G}, \overline{\mathbb{Q}}) \cong \operatorname{Hom}_{\Omega_{\mathbb{Q}}}(R(G), \overline{\mathbb{Q}}) .
$$

It is straightforward to verify that this isomorphism is the inverse of $\lambda_{G}$.

\section{The canonical fractional Galois ideal $\mathcal{J}_{E / F}^{r}$ in the abelian case}

\subsection{Definition of $\mathcal{J}_{E / F}^{r}$}

In this section we recall the canonical fractional Galois ideal introduced in 34 (see also [5, 31] and [33]). In [34 this was denoted merely by $\mathcal{J}_{E}^{r}$ but in this paper we shall need to keep track of the base field.

As in $\$ 2.2$, let $E / F$ be a Galois extension of number fields. Throughout this section we shall assume that the Stark conjecture of $\$ 2.2$ is true for all $E / F$ 
and that $G=\operatorname{Gal}(E / F)$ is abelian. Therefore, by Proposition 2.2, for each $r=0,-1,-2,-3, \ldots$ we have an element

$$
\mathcal{R}_{f_{E}^{r}} \in \operatorname{Hom}_{\Omega_{\mathbb{Q}}}\left(R(G), \overline{\mathbb{Q}}^{\times}\right) \cong \mathbb{Q}[G]^{\times}
$$

which depends upon the choice of a $\mathbb{Q}[G]$-isomorphism $f_{E}^{r}$ in $\$ 2.2$.

Let $\alpha \in \operatorname{End}_{\mathbb{Q}[G]}\left(Y_{r}(E)^{+} \otimes \mathbb{Q}\right)$ and extend this by the identity on the $(-1)$ eigenspace of complex conjugation $Y_{r}(E)^{-} \otimes \mathbb{Q}$ to give

$$
\alpha \oplus 1 \in \operatorname{End}_{\mathbb{Q}[G]}\left(Y_{r}(E) \otimes \mathbb{Q}\right) .
$$

Since $Y_{r}(E) \otimes \mathbb{Q}$ is free over $\mathbb{Q}[G]$, we may form the determinant

$$
\operatorname{det}_{\mathbb{Q}[G]}(\alpha \oplus 1) \in \mathbb{Q}[G] .
$$

In terms of the isomorphism of Proposition 2.2 , $\operatorname{det}_{\mathbb{Q}[G]}(\alpha \oplus 1)$ corresponds to the function which sends $\chi \in \widehat{G}$ to the determinant of the endomorphism of $e_{\chi} Y_{r}(E) \otimes \overline{\mathbb{Q}}$ induced by $\alpha \oplus 1$.

Following [34, Section 4.2] (see also [33, 31]), define $\mathcal{I}_{f_{E}^{r}}$ to be the (finitely generated) $\mathbb{Z}[1 / 2][G]$-submodule of $\mathbb{Q}[G]$ generated by all the elements $\operatorname{det}_{\mathbb{Q}[G]}(\alpha \oplus 1)$ satisfying the integrality condition

$$
\alpha \cdot f_{E}^{r}\left(K_{1-2 r}\left(\mathcal{O}_{E}\left[t^{ \pm 1}\right]\right)\right) \subseteq Y_{r}(E)
$$
by

Define $\mathcal{J}_{E / F}^{r}$ to be the finitely generated $\mathbb{Z}[1 / 2][G]$-submodule of $\mathbb{Q}[G]$ given

$$
\mathcal{J}_{E / F}^{r}=\mathcal{I}_{f_{E}^{r}} \cdot \tau\left(\mathcal{R}_{f_{E}^{r}}^{-1}\right)
$$

where $\tau$ is the automorphism of the group-ring induced by sending each $g \in G$ to its inverse.

Proposition 3.1 ([34, Prop.4.5]) Let E/F be a Galois extension of number fields with abelian Galois group $G$. Then, assuming that the Stark conjecture of 2.2 holds for $E / F$ for $r=0,-1,-2,-3, \ldots$, the finitely generated $\mathbb{Z}[1 / 2][G]$ submodule $\mathcal{J}_{E / F}^{r}$ of $\mathbb{Q}[G]$ just defined is independent of the choice of $f_{E}^{r}$.

\subsection{Naturality examples}

Given an extension $E / F$ of number fields satisfying the Stark conjecture at $s=0$ and a finite set of places $S$ of $F$ containing the infinite places, let $\mathcal{J}(E / F, S)$ denote the fractional Galois ideal as defined in [5], a slight modification of the one just defined so that we can take into account finite places. Let us consider the following situation: $\ell$ is an odd prime, $E_{n}=\mathbb{Q}\left(\zeta_{\ell^{n+1}}\right)$ for a primitive $\ell^{n+1}$ th root of unity $\zeta_{\ell^{n+1}}(n \geq 0)$, and $S=\{\infty, \ell\}$. The descriptions below of $\mathcal{J}\left(E_{n} / \mathbb{Q}, S\right)$ and $\mathcal{J}\left(E_{n}^{+} / \mathbb{Q}, S\right)$ are provided in [5, Section 4]:

$$
\begin{aligned}
\mathcal{J}\left(E_{n} / \mathbb{Q}, S\right) & =\frac{1}{2} e_{+} \operatorname{ann}_{\mathbb{Z}\left[G_{n}\right]}\left(\mathcal{O}_{E_{n}^{+}, S}^{\times} / \mathcal{E}_{n}^{+}\right) \oplus \mathbb{Z}\left[G_{n}\right] \theta_{E_{n} / \mathbb{Q}, S} \\
\mathcal{J}\left(E_{n}^{+} / \mathbb{Q}, S\right) & =\frac{1}{2} \operatorname{ann}_{\mathbb{Z}\left[G_{n}^{+}\right]}\left(\mathcal{O}_{E_{n}^{+}, S}^{\times} / \mathcal{E}_{n}^{+}\right)
\end{aligned}
$$


where $G_{n}=\operatorname{Gal}\left(E_{n} / \mathbb{Q}\right), G_{n}^{+}=\operatorname{Gal}\left(E_{n}^{+} / \mathbb{Q}\right), \mathcal{E}_{n}^{+}$is the $\mathbb{Z}\left[G_{n}^{+}\right]$-submodule of $\mathcal{O}_{E_{n}^{+}, S}^{\times}$generated by -1 and $\left(1-\zeta_{\ell^{n+1}}\right)\left(1-\zeta_{\ell^{n+1}}^{-1}\right)$, and $\theta_{E_{n} / \mathbb{Q}, S}$ is the Stickelberger element at $s=0$. Also, $e_{+}=\frac{1}{2}(1+c)$ is the plus-idempotent for complex conjugation $c \in G_{n}$.

It is immediate from these descriptions that the natural maps $\mathbb{Q}\left[G_{n}\right] \rightarrow$ $\mathbb{Q}\left[G_{n}^{+}\right], \mathbb{Q}\left[G_{n}\right] \rightarrow \mathbb{Q}\left[G_{n-1}\right]$ and $\mathbb{Q}\left[G_{n}^{+}\right] \rightarrow \mathbb{Q}\left[G_{n-1}^{+}\right]$give rise to a commutative diagram

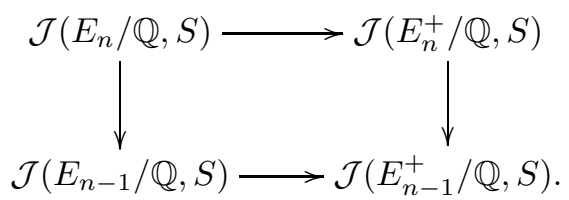

$\left(\mathcal{O}_{E_{n-1}^{+}, S}^{\times} / \mathcal{E}_{n-1}^{+}\right.$embeds into $\mathcal{O}_{E_{n}^{+}, S}^{\times} / \mathcal{E}_{n}^{+}$, and Stickelberger elements are well known (e.g. [18]) to map to each other in this way.)

Now suppose that $\ell \equiv 3 \bmod 4$, so that $E_{n}$ contains the imaginary quadratic field $F=\mathbb{Q}(\sqrt{-\ell})$. Again, letting $S_{F}$ consist of the infinite place of $F$ and the unique place above $\ell, \mathcal{J}\left(E_{n} / F, S_{F}\right)$ has a simple description. Indeed, if $H_{n}=\operatorname{Gal}\left(E_{n} / F\right)$, then

$$
\mathcal{J}\left(E_{n} / F, S_{F}\right)=\frac{1}{\mu_{n}} \operatorname{ann}_{\mathbb{Z}\left[H_{n}\right]}\left(\mathcal{O}_{E_{n}, S}^{\times} / \mathcal{E}_{n}\right)
$$

where $\mathcal{E}_{n}$ is generated over $\mathbb{Z}\left[H_{n}\right]$ by $\zeta_{\ell^{n+1}}$ and $\left(1-\zeta_{\ell^{n+1}}\right)^{\mu_{n} \tilde{\theta}_{n}}$. Here, $\mu_{n}=$ $\left|\mu\left(E_{n}\right)\right|$ and $\tilde{\theta}_{n}=\sum_{\sigma \in H_{n}} \zeta_{E_{n} / \mathbb{Q}, S}\left(0, \sigma^{-1}\right) \sigma \in \mathbb{Q}\left[H_{n}\right]$, a sort of "half Stickelberger element" obtained by keeping only those terms corresponding to elements in the index two subgroup $H_{n}$ of $G_{n}$. (Note that $\mu_{n} \tilde{\theta}_{n} \in \mathbb{Z}\left[H_{n}\right]$.) Comparing (3.2) and (3.4), we see without too much difficulty that

Proposition 3.2 The isomorphism $\Phi_{n}: \mathbb{Q}\left[H_{n}\right] \rightarrow \mathbb{Q}\left[G_{n}^{+}\right]$identifies $\mathcal{J}\left(E_{n} / F, S_{F}\right)$ with $2 \Phi_{n}\left(\tilde{\theta}_{n}\right) \mathcal{J}\left(E_{n}^{+} / \mathbb{Q}, S\right)$.

We now explain the above phenomena by proving some general relationships between the $\mathcal{J}_{E / F}^{r}$ under natural changes of extension.

\subsection{Behaviour under quotient maps $\operatorname{Gal}(L / F) \rightarrow \operatorname{Gal}(K / F)$}

Suppose that $F \subseteq K \subseteq L$ is a tower of number fields with $L / F$ abelian. The inclusion of $K$ into $L$ induces a homomorphism

$$
K_{1-2 r}\left(\mathcal{O}_{K}\left[t^{ \pm 1}\right]\right) \rightarrow K_{1-2 r}\left(\mathcal{O}_{L}\left[t^{ \pm 1}\right]\right) .
$$

When $r=0$

$$
\frac{K_{1}\left(\mathcal{O}_{K}\left[t^{ \pm 1}\right]\right)}{\text { Torsion }} \cong \mathcal{O}_{K}^{\times} /(\mu(K)) \oplus \mathbb{Z}\langle t\rangle
$$

maps injectively to the Galois invariants of $\mathcal{O}_{L}^{\times} /(\mu(L)) \oplus \mathbb{Z}\langle t\rangle$ sending $t$ to itself. For strictly negative $r$,

$$
\frac{K_{1-2 r}\left(\mathcal{O}_{K}\left[t^{ \pm 1}\right]\right)}{\text { Torsion }} \cong \frac{K_{1-2 r}\left(\mathcal{O}_{K}\right)}{\text { Torsion }}
$$


embeds into the $\operatorname{Gal}(L / K)$-invariants of $\frac{K_{1-2 r}\left(\mathcal{O}_{L}\left[t^{ \pm 1}\right]\right)}{\text { Torsion }}$. There is a homomorphism $Y_{r}(K) \rightarrow Y_{r}(L)$ which sends $n_{\sigma} \cdot \sigma$ to $n_{\sigma} \cdot\left(\sum_{\left(\sigma^{\prime} \mid F\right)=\sigma} \sigma^{\prime}\right)$ which is an isomorphism onto the $\operatorname{Gal}(L / K)$-invariants $Y_{r}(L)^{\operatorname{Gal}(L / K)}$. For $r=0,-1,-2,-3, \ldots$ there is a commutative diagram of regulators in $\$ 2.1$

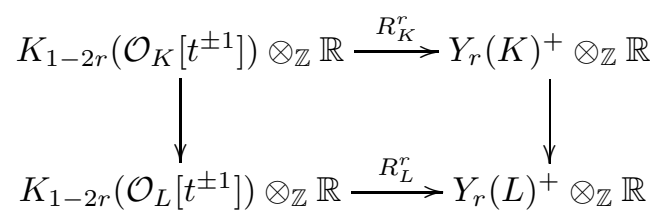

We may choose $f_{K}^{r}$ and $f_{L}^{r}$ as in 2.1 to make the corresponding diagram of $\mathbb{Q}$-vector spaces commute

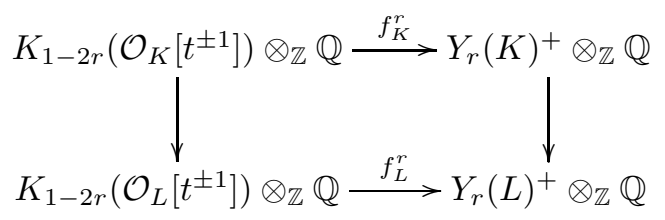

Let $V$ be a one-dimensional complex representation of $\operatorname{Gal}(K / F)$ and let $W=\operatorname{Inf}_{\operatorname{Gal}(K / F)}^{\operatorname{Gal}(L / F)}(V)$ denote the inflation of $V$. Then

$$
\begin{aligned}
& \operatorname{Hom}_{\operatorname{Gal}(L / F)}\left(W^{\vee}, Y_{r}(L)^{+} \otimes \mathbb{C}\right) \\
& =\operatorname{Hom}_{\operatorname{Gal}(L / F)}\left(W^{\vee},\left(Y_{r}(L)^{\operatorname{Gal}(L / K)}\right)^{+} \otimes \mathbb{C}\right) \\
& =\operatorname{Hom}_{\operatorname{Gal}(K / F)}\left(V^{\vee}, Y_{r}(K)^{+} \otimes \mathbb{C}\right)
\end{aligned}
$$

and these isomorphisms transport $\left(R_{L}^{r} \cdot\left(f_{L}^{r}\right)^{-1}\right)_{*}$ into $\left(R_{K}^{r} \cdot\left(f_{K}^{r}\right)^{-1}\right)_{*}$ by virtue of the above commutative diagrams. Furthermore, since the Artin $L$-function is invariant under inflation, $L_{F}^{*}(r, V)=L_{F}^{*}(r, W)$. On the other hand, the inflation homomorphism

$$
\operatorname{Inf}_{\operatorname{Gal}(K / F)}^{\operatorname{Gal}(L / F)}: R(\operatorname{Gal}(K / F)) \rightarrow R(\operatorname{Gal}(L / F))
$$

induces the canonical quotient map

$$
\pi_{L / K}: \mathbb{Q}[\operatorname{Gal}(L / F)]^{\times} \rightarrow \mathbb{Q}[\operatorname{Gal}(K / F)]^{\times}
$$

via the isomorphism of Proposition 2.2. Hence

$$
\pi_{L / K}\left(\mathcal{R}_{f_{L}^{r}}\right)=\mathcal{R}_{f_{K}^{r}} .
$$

Let $\alpha \in \operatorname{End}_{\mathbb{Q}[\operatorname{Gal}(L / F)]}\left(Y_{r}(L)^{+} \otimes \mathbb{Q}\right)$ satisfy the integrality condition of $\$ 3.1$

$$
\alpha \cdot f_{L}^{r}\left(K_{1-2 r}\left(\mathcal{O}_{L}\left[t^{ \pm 1}\right]\right)\right) \subseteq Y_{r}(L) .
$$

Extend this by the identity on the $(-1)$-eigenspace of complex conjugation $Y_{r}(L)^{-} \otimes \mathbb{Q}$ to give

$$
\alpha \oplus 1 \in \operatorname{End}_{\mathbb{Q}[\operatorname{Gal}(L / F)]}\left(Y_{r}(L) \otimes \mathbb{Q}\right) .
$$


The endomorphism $\alpha$ commutes with the action by $\operatorname{Gal}(L / K)$ so there is $\hat{\alpha} \in$ $\operatorname{End}_{\mathbb{Q}[\operatorname{Gal}(K / F)]}\left(Y_{r}(K)^{+} \otimes \mathbb{Q}\right)$ making the following diagram commute

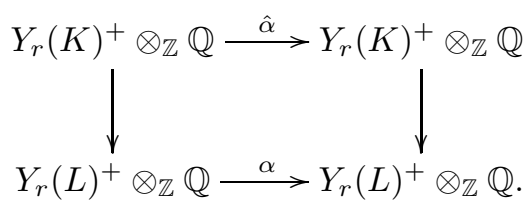

Therefore $\hat{\alpha}$ satisfies the integrality condition of $\$ 3.1$

$$
\hat{\alpha} \cdot f_{K}^{r}\left(K_{1-2 r}\left(\mathcal{O}_{K}\left[t^{ \pm 1}\right]\right)\right) \subseteq Y_{r}(K) .
$$

We may choose a $\mathbb{Z}[1 / 2][\operatorname{Gal}(K / F)]$ basis for $Y_{r}(K) \otimes \mathbb{Z}[1 / 2]$ consisting of embeddings $\sigma_{i}: K \rightarrow \mathbb{C}$ for $1 \leq i \leq m$. Let $\sigma_{i}^{\prime}$ be an embedding of $L$ which extends $\sigma_{i}$ for $1 \leq i \leq m$. Then a $\mathbb{Z}[1 / 2][\operatorname{Gal}(L / F)]$ basis for $Y_{r}(L) \otimes \mathbb{Z}[1 / 2]$ is given by $\left\{\sigma_{1}^{\prime}, \sigma_{2}^{\prime}, \ldots, \sigma_{m}^{\prime}\right\}$. The embedding of $Y_{r}(K)$ into $Y_{r}(L)$ is given by $\sigma_{i} \mapsto \sum_{g \in \operatorname{Gal}(L / K)} g\left(\sigma_{i}^{\prime}\right)$ which implies that the $m \times m$ matrix for $\hat{\alpha}$ with respect to the $\mathbb{Z}[1 / 2][\mathrm{Gal}(K / F)]$ basis of $\sigma_{i}$ 's is the image of the $m \times m$ matrix for $\alpha$ with respect to the $\mathbb{Z}[1 / 2][\operatorname{Gal}(L / F)]$ basis of $\sigma_{i}^{\prime}$ 's under the canonical surjection

$$
\mathbb{Q}[\operatorname{Gal}(L / F)] \rightarrow \mathbb{Q}[\operatorname{Gal}(K / F)]
$$

This discussion has established the following result.

Proposition 3.3 Suppose that $F \subseteq K \subseteq L$ is a tower of number fields with $L / F$ abelian. Then, in the notation of $\$ 3.1$, the canonical surjection

$$
\pi_{L / K}: \mathbb{Q}[\operatorname{Gal}(L / F)] \rightarrow \mathbb{Q}[\operatorname{Gal}(K / F)]
$$

satisfies

$$
\pi_{L / K}\left(\mathcal{J}_{L / F}^{r}\right) \subseteq \mathcal{J}_{K / F}^{r}
$$

Proposition 3.3 explains the existence of the maps in (3.3).

\subsection{Behaviour under inclusion maps $\operatorname{Gal}(L / K) \rightarrow \operatorname{Gal}(L / F)$}

As in 3.3 suppose that $F \subseteq K \subseteq L$ is a tower of number fields with $L / F$ abelian. The inclusion of $\operatorname{Gal}(L / K)$ into $\operatorname{Gal}(L / F)$ induces an inclusion of group-rings $\mathbb{Q}[\operatorname{Gal}(L / K)]$ into $\mathbb{Q}[\mathrm{Gal}(L / F)]$. In terms of the isomorphism of Proposition 2.2. as is easily seen by the formula, this homomorphism is induced by the restriction of representations

$$
\operatorname{Res}_{\operatorname{Gal}(L / K)}^{\operatorname{Gal}(L / F)}: R(\operatorname{Gal}(L / F)) \rightarrow R(\operatorname{Gal}(L / K)) .
$$


If $V$ is a complex representation of $\operatorname{Gal}(L / F)$ then

$$
\begin{aligned}
\mathcal{R}_{f_{L}^{r}}\left(\operatorname{Res}_{\operatorname{Gal}(L / K)}^{\operatorname{Gal}(L / F)}(V)\right) & =\frac{R\left(\operatorname{Res}_{\operatorname{Gal}(L / K)}^{\operatorname{Gal}(L / F)}(V), f_{L}^{r}\right)}{L_{K}^{*}\left(r, \operatorname{Res}_{\operatorname{Gal}(L / F)}^{\operatorname{Gal}(L / F)}(V)\right)} \\
& =\frac{R\left(\operatorname{Res}_{\operatorname{Gal}(L / K)}^{\operatorname{Gal}(L / F)}(V), f_{L}^{r}\right)}{L_{F}^{*}\left(r, \operatorname{Ind}_{\operatorname{Gal}(L / F)}^{\operatorname{Gal}(L / K)}\left(\operatorname{Res}_{\operatorname{Gal}(L / F)}^{\operatorname{Gal}(L / F)}(V)\right)\right)} \\
& =\frac{R\left(\operatorname{Res}_{\operatorname{Gal}(L / K)}^{\operatorname{Gal}(L / F)}(V), f_{L}^{r}\right)}{L_{F}^{*}\left(r, V \otimes \operatorname{Ind}_{\operatorname{Gal}(L / K)}^{\operatorname{Gal}(L / F)}(1)\right)} .
\end{aligned}
$$

If $W_{i} \in \widehat{\mathrm{Gal}}(L / F)$ for $1 \leq i \leq[K: F]$ is the set of one-dimensional representations which restrict to the trivial representation on $\operatorname{Gal}(L / K)$ then $\left.\operatorname{Ind}_{\operatorname{Gal}(L / K)}^{\operatorname{Gal}(L / F)}(1)\right)=\oplus_{i} W_{i}$. By Frobenius reciprocity

$$
\begin{aligned}
& \left.\operatorname{Hom}_{\operatorname{Gal}(L / K)}\left(\operatorname{Res}_{\operatorname{Gal}(L / K)}^{\operatorname{Gal}(L / F)}(V)^{\vee}, Y_{r}(L)^{+} \otimes \mathbb{C}\right)\right) \\
& \left.=\operatorname{Hom}_{\operatorname{Gal}(L / F)}\left(\oplus_{i}\left(V \otimes W_{i}\right)^{\vee}, Y_{r}(L)^{+} \otimes \mathbb{C}\right)\right)
\end{aligned}
$$

so that

$$
R\left(\operatorname{Res}_{\operatorname{Gal}(L / K)}^{\operatorname{Gal}(L / F)}(V), f_{L}^{r}\right)=\prod_{i} R\left(V \otimes W_{i}, f_{L}^{r}\right)
$$

and

$$
\mathcal{R}_{f_{L}^{r}}\left(\operatorname{Res}_{\operatorname{Gal}(L / K)}^{\operatorname{Gal}(L / F)}(V)\right)=\prod_{i} \mathcal{R}_{f_{L}^{r}}\left(V \otimes W_{i}\right) .
$$

Let $H \subseteq G$ be finite groups with $G$ abelian. It will suffice to consider the case in which $G / H$ is cyclic of order $n$ generated by $g H$. Let $W \otimes \mathbb{Q}$ be a free $\mathbb{Q}[G]$-module with basis $v_{1}, \ldots, v_{r}$. Then $W \otimes \mathbb{Q}$ is a free $\mathbb{Q}[H]$-module with basis $\left\{g^{a} v_{i} \mid 0 \leq a \leq n-1,1 \leq i \leq r\right\}$. Set $\mathcal{S}=\{0, \ldots, n-1\} \times\{1, \ldots, r\}$; then for $\underline{u}=(a, i) \in \mathcal{S}$, we set $e_{\underline{u}}=g^{a} v_{i}$. If $\tilde{\alpha} \in \operatorname{End}_{\mathbb{Q}[H]}(W \otimes \mathbb{Q})$ we may write

$$
\tilde{\alpha}\left(e_{\underline{w}}\right)=\sum_{\underline{u}} A_{\underline{u} \cdot \underline{w}} e_{\underline{u}}
$$

so that $A$ is an $n r \times n r$ matrix with entries in $\mathbb{Q}[H]$.

Now consider the induced $\mathbb{Q}[G]$-module $\operatorname{Ind}_{H}^{G}(W \otimes \mathbb{Q})$, which is a free $\mathbb{Q}[G]$ module on the basis $\left\{1 \otimes_{H} e_{\underline{u}} \mid \underline{u} \in \mathcal{S}\right\}$. Hence the $n r \times n r$ matrix, with entries in $\mathbb{Q}[G]$, for $1 \otimes_{H} \tilde{\alpha}$ with respect to this basis is the image of $A$ under the canonical inclusion of $\phi_{H, G}: \mathbb{Q}[H] \rightarrow \mathbb{Q}[G]$. In particular

$$
\phi_{H, G}\left(\operatorname{det}_{\mathbb{Q}[H]}(\tilde{\alpha})\right)=\operatorname{det}_{\mathbb{Q}[G]}\left(\mathbb{Q}[G] \otimes_{\mathbb{Q}[H]} \tilde{\alpha}\right)
$$

and, by induction on $[G: H]$, this relation is true for an arbitrary inclusion $H \subseteq G$ of finite abelian groups.

This discussion yields the following result: 
Proposition 3.4 Suppose that $F \subseteq K \subseteq L$ is a tower of number fields with $L / F$ abelian. Then, in the notation of \$3.1, the canonical inclusion

$$
\phi_{K / F}: \mathbb{Q}[\operatorname{Gal}(L / K)] \rightarrow \mathbb{Q}[\operatorname{Gal}(L / F)]
$$

maps $\mathcal{J}_{L / K}^{r}$ onto the $\mathbb{Z}[1 / 2][\operatorname{Gal}(L / K)]$-submodule

$\mathbb{Z}[1 / 2][\operatorname{Gal}(L / K)]\left\langle\operatorname{det}_{\mathbb{Q}[\operatorname{Gal}(L / F)]}\left(\mathbb{Q}[\operatorname{Gal}(L / F)] \otimes_{\mathbb{Q}[\operatorname{Gal}(L / K)]}(\alpha \oplus 1)\right) \tau\left(\hat{\mathcal{R}}_{f_{L}^{r}}\right)^{-1}\right\rangle$.

Here, in terms of Proposition [2.2, $\hat{\mathcal{R}}_{f_{L}^{r}} \in \mathbb{Q}[\operatorname{Gal}(L / F)]^{\times}$is given by

$$
\hat{\mathcal{R}}_{f_{L}^{r}}(V)=\mathcal{R}_{f_{L}^{r}}\left(V \otimes \operatorname{Ind}_{\operatorname{Gal}(L / K)}^{\operatorname{Gal}(L / F)}(1)\right)
$$

and $\alpha \in \operatorname{End}_{\mathbb{Q}[\operatorname{Gal}(L / K)]}\left(Y_{r}(L)^{+} \otimes \mathbb{Q}\right)$ runs through endomorphisms satisfying the integrality condition of $\$ 3.1$.

\subsection{Behaviour under fixed-point maps}

As in 3.3 suppose that $F \subseteq K \subseteq L$ is a tower of number fields with $L / F$ abelian. Let $e_{L / K}=[L: K]^{-1}\left(\sum_{y \in \operatorname{Gal}(L / K)} y\right)$ denote the idempotent associated with the subgroup $\operatorname{Gal}(L / K)$. There is a homomorphism of unital rings of the form

$$
\lambda_{K / F}: \mathbb{Q}[\operatorname{Gal}(K / F)] \rightarrow \mathbb{Q}[\operatorname{Gal}(L / F)]
$$

given, for $z \in \operatorname{Gal}(L / F)$, by the formula

$$
\lambda_{K / F}(z \operatorname{Gal}(L / K))=\left(1-e_{L / K}\right)+z \cdot e_{L / K} \in \mathbb{Q}[\operatorname{Gal}(L / F)] .
$$

From Proposition 2.2 it is easy to see that in terms of group characters

$$
\operatorname{Map}(\widehat{\operatorname{Gal}}(K / F), \overline{\mathbb{Q}}) \rightarrow \operatorname{Map}(\widehat{\operatorname{Gal}}(L / F), \overline{\mathbb{Q}})
$$

this sends a function $h$ on $\widehat{\operatorname{Gal}}(K / F)$ to the function $h^{\prime}$ given by

$$
h^{\prime}(\chi)= \begin{cases}h\left(\chi_{1}\right) & \text { if } \operatorname{Inf}_{\mathrm{Gal}(K / F)}^{\mathrm{Gal}(L / F)}\left(\chi_{1}\right)=\chi, \\ 1 & \text { otherwise. }\end{cases}
$$

Sending a complex representation $V$ of $\operatorname{Gal}(L / F)$ to its $\operatorname{Gal}(L / K)$-fixed points $V^{\operatorname{Gal}(L / K)}$ gives a homomorphism

$$
\text { Fix }: R(\operatorname{Gal}(L / F)) \rightarrow R(\operatorname{Gal}(K / F)) .
$$

In terms of one-dimensional respresentations (i.e. characters) the above condi-

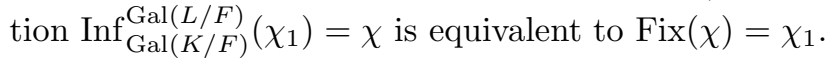

Let $V$ be a one-dimensional complex representation of $\operatorname{Gal}(L / F)$ fixed by $\operatorname{Gal}(L / K)$. Then we have isomorphisms of the form

$$
\begin{aligned}
& \operatorname{Hom}_{\operatorname{Gal}(L / F)}\left(\left(V^{\operatorname{Gal}(L / K)}\right)^{\vee}, Y_{r}(L)^{+} \otimes \mathbb{C}\right) \\
& =\operatorname{Hom}_{\operatorname{Gal}(K / F)}\left(V^{\vee},\left(Y_{r}(L)^{\operatorname{Gal}(L / K)}\right)^{+} \otimes \mathbb{C}\right) \\
& =\operatorname{Hom}_{\operatorname{Gal}(K / F)}\left(V^{\vee}, Y_{r}(K)^{+} \otimes \mathbb{C}\right)
\end{aligned}
$$


and, by invariance of $L$-functions under inflation, $L_{F}^{*}(r, V)=L_{F}^{*}\left(r, V^{\operatorname{Gal}(L / K)}\right)$. Therefore, by the discussion of 3.3 .

$$
\mathcal{R}_{f_{L}^{r}}(V)=\mathcal{R}_{f_{K}^{r}}\left(V^{\operatorname{Gal}(L / K)}\right) .
$$

On the other hand, if $V^{\operatorname{Gal}(L / K)}=0$ then $\mathcal{R}_{f_{K}^{r}}\left(V^{\operatorname{Gal}(L / K)}\right)=1$ since both $L_{F}^{*}(r, 0)$ and the determinant of the identity map of the trivial vector space are equal to one. This establishes the formula

$$
\lambda_{K / F}\left(\mathcal{R}_{f_{K}^{r}}\right)=\left(1-e_{L / K}\right)+\mathcal{R}_{f_{L}^{r}} \cdot e_{L / K} .
$$

Now consider an endomorphism

$$
\alpha \in \operatorname{End}_{\mathbb{Q}[\operatorname{Gal}(K / F)]}\left(Y_{r}(K)^{+} \otimes \mathbb{Q}\right)
$$

satisfying the integrality condition of $\$ 3.1$

$$
\alpha f_{r, K}\left(K_{1-2 r}\left(\mathcal{O}_{K}\left[t^{ \pm 1}\right]\right)\right) \subseteq Y_{r}(K)^{+} \cong\left(Y_{r}(L)^{+}\right)^{\operatorname{Gal}(L / K)} .
$$

Let $v_{1}, v_{2}, \ldots, v_{d}$ be a $\mathbb{Z}[1 / 2][\operatorname{Gal}(L / F)]$-basis of $Y_{r}(L)[1 / 2]$ so that a $\mathbb{Z}[1 / 2][\operatorname{Gal}(K / F)]$-basis of the subspace $\left(Y_{r}(L)^{+}\right)^{\operatorname{Gal}(L / K)}[1 / 2] \cong Y_{r}(K)[1 / 2]$ is given by $\left\{\left(\sum_{y \in \operatorname{Gal}(L / K)} y\right) v_{i} \mid 1 \leq i \leq d\right\}$. To construct the generators of $\mathcal{J}_{K / F}^{r}$, as in 3.1 we must calculate the determinant of $\alpha \oplus 1$ on $Y_{r}(K)^{+} \otimes \mathbb{Q} \oplus$ $Y_{r}(K)^{-} \otimes \mathbb{Q}=Y_{r}(K) \otimes \mathbb{Q}$ with respect to the basis $\left\{\left(\sum_{y \in \operatorname{Gal}(L / K)} y\right) v_{i}\right\}$ and divide by $\tau\left(\mathcal{R}_{f_{K}^{r}}\right)$.

Let $\hat{\alpha} \in \operatorname{End}_{\mathbb{Q}[\operatorname{Gal}(L / F)]}\left(Y_{r}(L) \otimes \mathbb{Q}\right)$ be given by $\alpha$ on $Y_{r}(L)^{\operatorname{Gal}(L / F)} \otimes \mathbb{Q}$ and the identity on $\left(1-e_{L / K}\right) Y_{r}(L) \otimes \mathbb{Q}$. Hence $\hat{\alpha}$ satisfies the integrality condition

$$
\hat{\alpha} \cdot f_{L}^{r}\left(K_{1-2 r}\left(\mathcal{O}_{L}\left[t^{ \pm 1}\right]\right)\right)^{\operatorname{Gal}(L / F)} \subseteq Y_{r}(L)^{\operatorname{Gal}(L / F)},
$$

because, as in $33.3 . f_{K}^{r}$ may be assumed to extend to $f_{L}^{r}$. Therefore

$$
e_{L / K} \frac{\operatorname{det}(\hat{\alpha})}{\tau\left(\mathcal{R}_{f_{L}^{r}}\right)} \in e_{L / K} \mathcal{J}_{L / F}^{r} \subset \mathbb{Q}[\operatorname{Gal}(L / F)] .
$$

On the other hand it is clear that $\lambda_{K / F}(\operatorname{det}(\alpha \oplus 1))=\operatorname{det}(\hat{\alpha})$.

This discussion has established the following result.

Proposition 3.5 Suppose that $F \subseteq K \subseteq L$ is a tower of number fields with $L / F$ abelian and let

$$
\lambda_{K / F}: \mathbb{Q}[\operatorname{Gal}(K / F)] \rightarrow \mathbb{Q}[\operatorname{Gal}(L / F)]
$$

denote the unital ring homomorphism of \$3.5. Then

$$
\lambda_{K / F}\left(\mathcal{J}_{K / F}^{r}\right) \subseteq\left(1-e_{L / K}\right) \mathbb{Q}[\operatorname{Gal}(L / F)]+e_{L / K} \mathcal{J}_{L / F}^{r}
$$




\subsection{Behaviour under corestriction maps}

As in 3.3 suppose that $F \subseteq K \subseteq L$ is a tower of number fields with $L / F$ abelian. There is an additive homomorphism of the form

$$
\iota_{K / F}: \mathbb{Q}[\operatorname{Gal}(L / F)] \rightarrow \mathbb{Q}[\operatorname{Gal}(L / K)]
$$

called the transfer or corestriction map. In terms of Proposition2.2 it is induced by the induction of representations

$$
\operatorname{Ind}_{\operatorname{Gal}(L / K)}^{\operatorname{Gal}(L / F)}: R(\operatorname{Gal}(L / K)) \rightarrow R(\operatorname{Gal}(L / F)) .
$$

That is, the image $\iota_{K / F}(h)$ of $h \in \operatorname{Hom}_{\Omega_{\mathbb{Q}}}(R(\operatorname{Gal}(L / F)), \overline{\mathbb{Q}})$ is given by

$$
\iota_{K / F}(h)(V)=h\left(\operatorname{Ind}_{\operatorname{Gal}(L / K)}^{\operatorname{Gal}(L / F)}(V)\right) .
$$

By Frobenius reciprocity, for each $V \in R(\mathrm{Gal})(L / K))$ there is an isomorphism

$$
\begin{aligned}
& \operatorname{Hom}_{\operatorname{Gal}(L / F)}\left(\left(\operatorname{Ind}_{\mathrm{Gal}(L / F)}^{\operatorname{Gal}(L / F)}\right)^{\vee}, Y_{r}(L)^{+} \otimes \mathbb{C}\right) \\
& =\operatorname{Hom}_{\operatorname{Gal}(L / K)}\left(V^{\vee}, Y_{r}(L)^{+} \otimes \mathbb{C}\right) .
\end{aligned}
$$

Also $L_{F}^{*}\left(r, \operatorname{Ind}_{\operatorname{Gal}(L / K)}^{\operatorname{Gal}(L / F)}(V)\right)=L_{K}^{*}(r, V)$ so that

$$
\iota_{K / F}\left(\mathcal{R}_{f_{L}^{r}}\right)=\mathcal{R}_{f_{L}^{r}} .
$$

Now consider an endomorphism

$$
\alpha \in \operatorname{End}_{\mathbb{Q}[\operatorname{Gal}(L / F)]}\left(Y_{r}(L)^{+} \otimes \mathbb{Q}\right)
$$

satisfying the integrality condition of 3.1

$$
\alpha f_{r, L}\left(K_{1-2 r}\left(\mathcal{O}_{L}\left[t^{ \pm 1}\right]\right)\right) \subseteq Y_{r}(L)^{+} .
$$

Then it is straightforward to see from Proposition 2.2 that the determinant of $\alpha \oplus 1$ as a map of $\mathbb{Q}[\operatorname{Gal}(L / F)]$-modules $\operatorname{det}_{\mathbb{Q}[\operatorname{Gal}(L / F)]}(\alpha \oplus 1)$ is mapped to $\operatorname{det}_{\mathbb{Q}[\operatorname{Gal}(L / K)]}(\alpha \oplus 1)$, the determinant of $\alpha \oplus 1$ as $\mathbb{Q}[\operatorname{Gal}(L / K)]$-modules.

This discussion has established the following result. (This result has a problem. See Section 1.1.)

Proposition 3.6 Suppose that $F \subseteq K \subseteq L$ is a tower of number fields with $L / F$ abelian, and let

$$
\iota_{K / F}: \mathbb{Q}[\operatorname{Gal}(L / F)] \rightarrow \mathbb{Q}[\operatorname{Gal}(L / K)]
$$

denote the additive homomorphism of \$3.6. Then

$$
\iota_{K / F}\left(\mathcal{J}_{L / F}^{r}\right) \subseteq \mathcal{J}_{L / K}^{r} .
$$




\section{7}

We can now explain the second example in 3.2 , i.e. Proposition 3.2 Let us work more generally to begin with. $E$ and $F$ can be any number fields, and we suppose we have a diagram

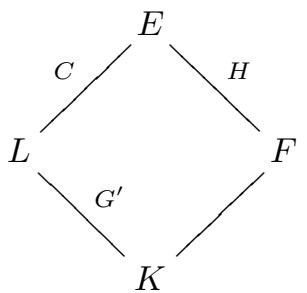

satisfying the following: $E / K$ is Galois (though not necessarily abelian), $L F=$ $E, L \cap F=K$, the extension $L / K$ is abelian (and hence so is $E / F$ ), and $L / K$ and $E / F$ satisfy the Stark conjecture. We let $G=\operatorname{Gal}(E / K)$, and the Galois groups of the other Galois extensions are marked in the diagram. We observe that $C$ need not be abelian here.

Owing to the natural isomorphism $G / C \rightarrow H$, each character $\psi \in \widehat{H}$ extends to a unique one-dimensional representation $\widehat{\psi}: G \rightarrow \mathbb{C}^{\times}$which is trivial on $C$. Denote by $\operatorname{ch}(G)$ the set of irreducible characters of $G$. Then having chosen a $\mathbb{Q}[G]$-module isomorphism $f$ as in $\$ 2.2$, we can define an element $\Omega^{f} \in \mathbb{C}[H]^{\times}$ by

$$
\Omega^{f}=\prod_{\chi \in \operatorname{ch}(G) \backslash\{1\}}\left(\sum_{\psi \in \widehat{H}} \mathcal{R}_{E / K}^{f}(\chi \widehat{\psi})^{d_{\chi}} e_{\psi}\right),
$$

where for a character $\chi$ of $G, d_{\chi}$ is the multiplicity of the trivial character of $H$ in $\operatorname{Res}_{H}^{G}(\chi)$. We have opted to denote by $\mathcal{R}_{E / K}^{f}$ the group-ring element $\mathcal{R}_{f_{E}}$ defined in 3.1, to emphasize which extension is being considered.

The following lemma shows that the group-ring element $\mathcal{R}_{E / F}^{f}$ for the extension $E / F$ is related, via $\Omega^{f}$, to the corresponding element for the extension $L / K$.

Lemma 3.7 $\Omega^{f}$ has rational coefficients, and the image of $\mathcal{R}_{E / F}^{f}$ under the isomorphism $\Phi: \mathbb{Q}[H] \rightarrow \mathbb{Q}\left[G^{\prime}\right]$ is

$$
\mathcal{R}_{L / K}^{f^{\prime}} \Phi\left(\Omega^{f}\right)
$$

where $f^{\prime}$ is the $\mathbb{Q}\left[G^{\prime}\right]$-module isomorphism making diagram (3.5) commute.

The proof of the lemma is little more than a combination of $\$ 3.3$ and $\$ 3.6$.

In the situation of Proposition 3.2 (with $L=E^{+}$and $K=\mathbb{Q}$ now) we find that the element $2 \tilde{\theta}$ occurring there is just $\tau\left(\Omega^{f}\right)^{-1}$ (for any choice of $f$ in this 
case). Indeed, let $\rho \in \widehat{G}$ be the unique non-trivial character extending the trivial character of $H$. Then the only $\chi \in \operatorname{ch}(G) \backslash\{1\}$ with $d_{\chi} \neq 0$ is $\rho$, and $d_{\rho}=1$, so

$$
\begin{aligned}
& \Omega^{f}=\sum_{\psi \in \widehat{H}} \mathcal{R}_{E / \mathbb{Q}}^{f}(\rho \widehat{\psi}) e_{\psi} \\
& =\sum_{\substack{\psi \in \widehat{G} \\
\psi \text { even }}} \mathcal{R}_{E / \mathbb{Q}}^{f}(\rho \psi) e_{\left.\psi\right|_{H}} .
\end{aligned}
$$

However, for $\psi$ even, $\rho \psi$ is odd so that $\mathcal{R}^{f}(\rho \psi)=L_{E / \mathbb{Q}, S}(0, \rho \psi)^{-1}$. Using the easily verified fact that $(1-c) \tilde{\theta}=\theta_{E / \mathbb{Q}, S}$, where $c \in G$ is complex conjugation, we see that $L_{E / \mathbb{Q}, S}(0, \rho \psi)=\left.2 \psi\right|_{H}(\tau \tilde{\theta})$, from which the assertion follows.

Applying Lemma 3.7 now justifies the appearance of $2 \Phi_{n}\left(\tilde{\theta}_{n}\right)$ in Proposition 3.2 .

\section{The passage to non-abelian groups}

\section{1}

In this section we shall use the Explicit Brauer Induction constructions of [32, pp.138-147] to pass from finite abelian Galois groups to the non-abelian case.

Let $G$ be a finite group and consider the additive homomorphism

$$
\sum_{H \subseteq G} \operatorname{Ind}_{H}^{G} \operatorname{Inf}_{H^{\mathrm{ab}}}^{H}: \oplus_{H \subseteq G} R\left(H^{\mathrm{ab}}\right) \rightarrow R(G) .
$$

Let $N \triangleleft G$ be a normal subgroup and let $\pi: G \rightarrow G / N$ denote the quotient homomorphism.

Define a homomorphism

$$
\alpha_{G, N}: \oplus_{J \subseteq G / N} R\left(J^{\mathrm{ab}}\right) \rightarrow \oplus_{H \subseteq G} R\left(H^{\mathrm{ab}}\right)
$$

to be the homomorphism which sends the $J$-component $R\left(J^{\mathrm{ab}}\right)$ to the $H=$ $\pi^{-1}(J)$-component $R\left(\pi^{-1}(J)^{\mathrm{ab}}\right)$ via the map

$$
\operatorname{Inf}_{J^{\mathrm{ab}}}^{\pi^{-1}(J)^{\mathrm{ab}}}\left(R\left(J^{\mathrm{ab}}\right)\right) \rightarrow R\left(\pi^{-1}(J)^{\mathrm{ab}}\right) .
$$

Lemma 4.1 In the notation of \$4.1 the following diagram commutes:




Proof. Since the kernel of $\pi^{-1}(J) \rightarrow J$ and that of $\pi: G \rightarrow G / N$ coincide, both being equal to $N$, we have

$$
\operatorname{Inf}_{G / N}^{G} \operatorname{Ind}_{J}^{G / N}=\operatorname{Ind}_{\pi^{-1}(J)}^{G} \operatorname{Inf}_{J}^{\pi^{-1}(J)} .
$$

Therefore, given a character $\phi: J^{\mathrm{ab}} \rightarrow \overline{\mathbb{Q}}^{\times}$in the $J$-coordinate, we have

$$
\begin{aligned}
\operatorname{Ind}_{\pi^{-1}(J)}^{G} \operatorname{Inf}_{\pi^{-1}(J)^{\text {ab }}}^{\pi^{-1}(J), N}(\phi) & =\operatorname{Ind}_{\pi^{-1}(J)}^{G} \operatorname{Inf}_{\pi^{-1}(J)}^{\pi^{-1}(J)} \operatorname{Inf}_{J \text { ab }}^{\pi^{-1}(J)^{\mathrm{ab}}}(\phi) \\
& =\operatorname{Ind}_{\pi^{-1}(J)}^{G} \operatorname{Inf}_{J}^{\pi^{-1}(J)} \operatorname{Inf}_{J^{\mathrm{ab}}}^{J}(\phi) \\
& =\operatorname{Inf}_{G / N}^{G} \operatorname{Ind}_{J}^{G / N} \operatorname{Inf}_{J^{\text {ab }}}^{J}(\phi),
\end{aligned}
$$

as required.

\section{2}

The homomorphism of 4.1 is invariant under group conjugation and therefore induces an additive homomorphism of the form

$$
B_{G}:\left(\oplus_{H \subseteq G} R\left(H^{\mathrm{ab}}\right)\right)_{G} \rightarrow R(G)
$$

where $X_{G}$ denotes the coinvariants of the conjugation $G$-action. This homomorphism is a split surjection whose right inverse is given by the Explicit Brauer Induction homomorphism

$$
A_{G}: R(G) \rightarrow\left(\oplus_{H \subseteq G} R\left(H^{\mathrm{ab}}\right)\right)_{G}
$$

constructed in [32, Section 4.5.16]. We shall be interested in the dual homomorphisms ([32, Section 4.5.20])

$$
B_{G}^{*}: \operatorname{Hom}_{\Omega_{\mathbb{Q}}}(R(G), \overline{\mathbb{Q}}) \rightarrow\left(\oplus_{H \subseteq G} \operatorname{Hom}_{\Omega_{\mathbb{Q}}}\left(R\left(H^{\mathrm{ab}}\right), \overline{\mathbb{Q}}\right)\right)^{G}
$$

and

$$
A_{G}^{*}:\left(\oplus_{H \subseteq G} \operatorname{Hom}_{\Omega_{\mathbb{Q}}}\left(R\left(H^{\mathrm{ab}}\right), \overline{\mathbb{Q}}\right)\right)^{G} \rightarrow \operatorname{Hom}_{\Omega_{\mathbb{Q}}}(R(G), \overline{\mathbb{Q}})
$$

where $X^{G}$ denotes the subgroup of $G$-invariants.

As in [32, Def.4.5.4], denote by $\mathbb{Q}\{G\}$ the rational vector space whose basis consists of the conjugacy classes of $G$. There is an isomorphism ([32, Prop.4.5.14])

$$
\psi: \mathbb{Q}\{G\} \stackrel{\cong}{\rightrightarrows} \operatorname{Hom}_{\Omega_{\mathbb{Q}}}(R(G), \overline{\mathbb{Q}})
$$

given by the formula $\psi\left(\sum_{\gamma} m_{\gamma} \gamma\right)(\rho)=\sum_{\gamma} m_{\gamma} \operatorname{Trace}(\rho(\gamma))$.

When $G$ is abelian, we have $\mathbb{Q}\{G\}=\mathbb{Q}[G]$ and under the identification

$$
\operatorname{Hom}_{\Omega_{\mathbb{Q}}}(R(G), \overline{\mathbb{Q}})=\operatorname{Map}_{\Omega_{\mathbb{Q}}}(\widehat{G}, \overline{\mathbb{Q}})
$$

of Proposition 2.2 we have $\psi(g)=(\chi \mapsto \chi(g))$, which is a ring isomorphism inverse to $\lambda_{G}$. 


\section{$5 \quad \mathcal{J}_{E / F}^{r}$ in general}

Let $G$ denote the Galois group of a finite Galois extension $E / F$ of number fields. Hence each subgroup of $G$ has the form $H=\operatorname{Gal}\left(E / E^{H}\right)$, whose abelianization is $H^{\mathrm{ab}}=\operatorname{Gal}\left(E^{[H, H]} / E^{H}\right)$ where $[H, H]$ is the commutator subgroup of $H$. For each integer $r=0,-1,-2,-3, \ldots$, we have the canonical fractional Galois ideal $\mathcal{J}_{E^{[H, H]} / E^{H}}^{r} \subseteq \mathbb{Q}\left[H^{\mathrm{ab}}\right]$ as defined in $\$ 3.1$

Definition 5.1 In the notation of $\$ 5$, define a subgroup $\mathcal{J}_{E / F}^{r}$ of $\mathbb{Q}\{G\}$ by

$$
\mathcal{J}_{E / F}^{r}=\left(B_{G}^{*}\right)^{-1}\left(\oplus_{H \subseteq G} \mathcal{J}_{E^{[H, H]} / E^{H}}^{r}\right) .
$$

Lemma 5.2 In $\$ 5$ and Definition [5.1, when $G=\operatorname{Gal}(E / F)$ is abelian then $\mathcal{J}_{E / F}^{r}$ coincides with the canonical fractional Galois ideal of 3.1.

Proof. The $H$-component of $B_{G}^{*}$ has the form

$$
\mathbb{Q}[\operatorname{Gal}(E / F)] \stackrel{i_{E^{H} / F}}{\rightarrow} \mathbb{Q}\left[\operatorname{Gal}\left(E / E^{H}\right)\right] \stackrel{\pi_{E / E^{[H, H]}}}{\rightarrow} \mathbb{Q}\left[\operatorname{Gal}\left(E^{[H, H]} / E^{H}\right)\right]
$$

which maps $\mathcal{J}_{E / F}^{r}$ to $\mathcal{J}_{E^{[H, H]} / E^{H}}^{r}$ by Proposition 3.3 and Proposition 3.6 so that

$$
\mathcal{J}_{E / F}^{r} \subseteq\left(B_{G}^{*}\right)^{-1}\left(\oplus_{H \subseteq G} \mathcal{J}_{E[H, H]}^{r} / E^{H}\right) .
$$

On the other hand, the $G$-component of $B_{G}^{*}$ is the identity map from $\mathbb{Q}[G]$ to itself. Therefore if $z \in \mathbb{Q}[G] \backslash \mathcal{J}_{E / F}^{r}$ then $B_{G}^{*}(z) \notin \oplus_{H \subseteq G} \mathcal{J}_{E^{[H, H]} / E^{H}}^{r}$, as required.

Proposition 5.3 Suppose that $F \subseteq K \subseteq L$ is a tower of finite extensions of number fields with $L / F$ and $K / F$ Galois. Then, for $r=0,-1,-2,-3, \ldots$, the canonical homomorphism

$$
\pi_{L / K}: \mathbb{Q}\{\operatorname{Gal}(L / F)\} \rightarrow \mathbb{Q}\{\operatorname{Gal}(K / F)\}
$$

satisfies $\pi_{L / K}\left(\mathcal{J}_{E / F}^{r}\right) \subseteq \mathcal{J}_{K / F}^{r}$.

Proof. This follows immediately from Proposition 3.3, Lemma 4.1 and Definition 5.1

Definition 5.4 Let $F$ be a number field and $L / F$ a (possibly infinite) Galois extension with Galois group $G=\operatorname{Gal}(L / F)$. For $r=0,-1,-2,-3, \ldots$ define $\mathcal{J}_{E / F}^{r}$ to be the abelian group

$$
\mathcal{J}_{E / F}^{r}=\lim _{\leftarrow H} \mathcal{J}_{L^{H} / F}^{r},
$$

where $H$ runs through the open normal subgroups of $G$. 


\section{$6 \mathcal{J}_{E / F}^{r}$ and the annihilation of $H_{\text {êt }}^{2}\left(\operatorname{Spec}\left(\mathcal{O}_{L, S}\right), \mathbb{Z}_{\ell}(1-r)\right)$}

\section{1}

Let $\ell$ be an odd prime. We continue to assume the Stark conjecture as stated in $\$ 2.2$ for $r=0,-1,-2,-3, \ldots$. Replacing $\mathbb{Q}$ by $\mathbb{Q}_{\ell}$ in 3.1 and Definition 5.1 we may associate a finitely generated $\mathbb{Z}_{\ell}$-submodule of $\mathbb{Q}_{\ell}\{\operatorname{Gal}(E / F)\}$, again denoted by $\mathcal{J}_{E / F}^{r}$, to any finite extension $E / F$ of number fields.

In this section we are going to explain a conjectural procedure to pass from $\mathcal{J}_{E / F}^{r}$ to the construction of elements in the annihilator ideal of the étale cohomology of the ring of $S$-integers of $E$,

$$
\operatorname{ann}_{\mathbb{Z}_{\ell}[G(E / F)]}\left(H_{\text {ét }}^{2}\left(\operatorname{Spec}\left(\mathcal{O}_{E, S(E)}\right), \mathbb{Z}_{\ell}(1-r)\right)\right),
$$

where $S$ denotes a finite set of primes of $F$ including all archimedean primes and all finite primes which ramify in $E / F$, and $S(E)$ denotes all the primes of $E$ over those in $S$. This conjectural procedure was first described in [34, Thm.8.1].

We shall restrict ourselves to the case when $r=-1,-2,-3, \ldots$ In several ways this is a simplification over the case when $r=0$. In this case $H_{\text {ét }}^{1}\left(\operatorname{Spec}\left(\mathcal{O}_{E, S(E)}\right), \mathbb{Z}_{\ell}(1-r)\right)$ is independent of $S(E)$, while it is related to the group of $S(E)$-units when $r=0$. Also, when $r \leq-1, H_{\text {ét }}^{2}\left(\operatorname{Spec}\left(\mathcal{O}_{E, S(E)}\right), \mathbb{Z}_{\ell}(1-\right.$ $r)$ ) is a subgroup of the corresponding cohomology group when $S(E)$ is enlarged

to $S^{\prime}(E)$, but when $r=0$ the class-group of $\mathcal{O}_{E, S^{\prime}(E)}$ is a quotient of that of $\mathcal{O}_{E, S(E)}$. Furthermore (see [5], [36]), there are subtleties concerning whether or not to use the $S$-modified $L$-function in $\$ 2$ when $r=0$, while for $r \leq-1$ this is immaterial.

When $r=0$ the annihilator procedure is similar to the other cases but the additional complications have prompted us to omit this case.

Write $G=\operatorname{Gal}(E / F)$, and for each subgroup $H=\operatorname{Gal}\left(E / E^{H}\right) \subseteq G$ let $S\left(E^{H}\right)$ denote the set of primes of $E^{H}$ above those of $S$. Then $H^{\overline{\mathrm{ab}}}=$ $\operatorname{Gal}\left(E^{[H, H]} / E^{H}\right)$ where $[H, H]$ denotes the commutator subgroup of $H$. The following conjecture originated in $31,33,34$.

Conjecture 6.1 In the notation of $\$ 6.1$, when $r=-1,-2,-3, \ldots$,

(i) Integrality:

$$
\mathcal{J}_{E^{[H, H]} / E^{H}}^{r} \cdot \operatorname{ann}_{\mathbb{Z}_{\ell}\left[H^{\mathrm{ab}}\right]}\left(\operatorname{Tors} H_{\text {ét }}^{1}\left(\operatorname{Spec}\left(\mathcal{O}_{E^{[H, H]}, S}\right), \mathbb{Z}_{\ell}(1-r)\right)\right) \subseteq \mathbb{Z}_{\ell}\left[H^{\mathrm{ab}}\right] .
$$

\section{(ii) Annihilation:}

$$
\begin{aligned}
& \mathcal{J}_{E^{[H, H]} / E^{H}}^{r} \cdot \operatorname{ann}_{\mathbb{Z}_{\ell}\left[H^{\mathrm{ab}}\right]}\left(\operatorname{Tors} H_{\text {ét }}^{1}\left(\operatorname{Spec}\left(\mathcal{O}_{E^{[H, H]}, S}\right), \mathbb{Z}_{\ell}(1-r)\right)\right) \\
& \subseteq \operatorname{ann}_{\mathbb{Z}_{\ell}\left[H^{\mathrm{ab}}\right]}\left(H_{\text {ét }}^{2}\left(\operatorname{Spec}\left(\mathcal{O}_{E^{[H, H]}, S}\right), \mathbb{Z}_{\ell}(1-r)\right)\right) .
\end{aligned}
$$

(We have adopted the shorthand: $\mathcal{O}_{E^{[H, H]}, S}=\mathcal{O}_{E^{[H, H]}, S\left(E^{[H, H]}\right)}$.) 


\subsection{Evidence}

Part (i) of Conjecture 6.1 is analogous to the Stickelberger integrality, which is described in [34, Section 2.2]. Stickelberger integrality was proven in certain totally real cases in [21, 9, 8, 14, for $r=0$. In general, when $r=0$, it is part of the Brumer conjecture [4]. The novelty of part (ii) of Conjecture 6.1. when it was introduced in [33] and [34, was the annihilator prediction when the $L$-function vanishes at $s=r$. For the part of the fractional ideal corresponding to characters whose $L$-functions are non-zero at $s=r$, generated by the higher Stickelberger element at $s=r$, part (ii) is the conjecture of [12].

Let us consider the cyclotomic example $\mathcal{J}_{L / \mathbb{Q}}^{r}(r<0)$ when $L=\mathbb{Q}(\zeta)$ for some root of unity $\zeta$, and suppose $\ell$ is an odd prime dividing the order of $\zeta$. In this case, $\mathcal{J}_{L / \mathbb{Q}}^{r}$ splits into plus and minus parts for complex conjugation, i.e.

$$
\mathcal{J}_{L / \mathbb{Q}}^{r}=e_{+}^{r} \mathcal{J}_{L / \mathbb{Q}}^{r} \oplus e_{-}^{r} \mathcal{J}_{L / \mathbb{Q}}^{r},
$$

where $e_{+}^{r}=\frac{1}{2}\left(1+(-1)^{r} c\right), e_{-}^{r}=\frac{1}{2}\left(1-(-1)^{r} c\right)$ and $c \in G=\operatorname{Gal}(L / \mathbb{Q})$ is complex conjugation. By the proof of [34, Theorem 6.1], $e_{-}^{r} \mathcal{J}_{L / \mathbb{Q}}^{r}$ is generated by the Stickelberger element $\theta_{L / \mathbb{Q}, S}(r)$ defined in terms of $L$-function values at $s=r$. However, by [14,

$$
\operatorname{ann}_{\mathbb{Z}_{\ell}[G]}\left(\operatorname{Tors}\left(H_{\text {ét }}^{1}\left(\operatorname{Spec} \mathcal{O}_{L, S}, \mathbb{Z}_{\ell}(1-r)\right)\right)\right) \theta_{L / \mathbb{Q}, S}(r) \subseteq \mathbb{Z}_{\ell}[G] .
$$

Further, the proof of [34, Theorem 7.6] shows that $e_{+}^{r} \mathcal{J}_{L / \mathbb{Q}}^{r} \subseteq \mathbb{Z}_{\ell}[G]$. In fact, [34. Theorem 6.1] also shows that part (ii) of Conjecture 6.1 holds in this case (with $E=\mathbb{Q}$ and $H=G$ ), the intersection " $\cap \mathbb{Z}_{\ell}[G]$ " found in the statement of that theorem being unnecessary.

Turning now to the case $r=0$, with the field $E_{n}$ as in 3.2 we have a similar scenario for $\mathcal{J}\left(E_{n} / \mathbb{Q}, S\right)$, where $S=\{\infty, \ell\}$. Indeed, we see from (3.1) that $\mathcal{J}\left(E_{n} / \mathbb{Q}, S\right)$ again splits into plus and minus parts, with the minus part being generated by the Stickelberger element $\theta_{E_{n} / \mathbb{Q}, S}$ defined at $s=0$. Stickelberger's theorem then implies that

$$
\operatorname{ann}_{\mathbb{Z}_{\ell}\left[G_{n}\right]}\left(\mu\left(E_{n}\right)\right) e_{-} \mathcal{J}\left(E_{n} / \mathbb{Q}, S\right) \subseteq \mathbb{Z}_{\ell}\left[G_{n}\right],
$$

and $e_{+} \mathcal{J}\left(E_{n} / \mathbb{Q}, S\right)$ is already in $\mathbb{Z}_{\ell}\left[G_{n}\right]$. The roles of the plus and minus parts of $\mathcal{J}\left(E_{n} / \mathbb{Q}, S\right)$ will become clear in $\$ 6.2 .1$ below.

\subsubsection{An Iwasawa-theoretic example}

(3.1) can be used to provide an example of the relationship of $\mathcal{J}\left(E_{n} / \mathbb{Q}, S\right)$ to Iwasawa theory, with an inverse limit of the $\mathcal{J}\left(E_{n} / \mathbb{Q}, S\right)$ over $n$ giving rise, in a suitable way, to Fitting ideals of both the plus and minus parts of an inverse limit of class-groups (Proposition 6.2). Given $n \geq 0$, let $\mathbb{Q}^{(n)} / \mathbb{Q}$ be the degree $\ell^{n}$ subextension of the (unique) $\mathbb{Z}_{\ell}$-extension $\mathbb{Q}^{(\infty)}$ of $\mathbb{Q}$. We then have the field 
diagram

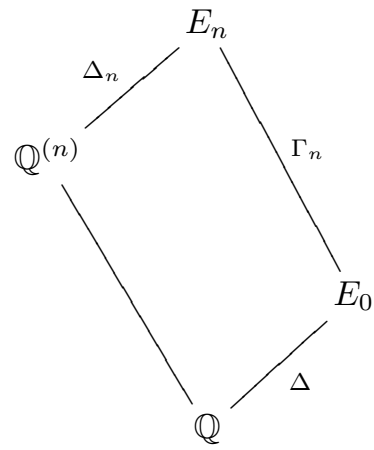

in which $\mathbb{Q}^{(n)} \cap E_{0}=\mathbb{Q}$ and $\mathbb{Q}^{(n)} E_{0}=E_{n}$, so that the Galois group $G_{n}=$ $\operatorname{Gal}\left(E_{n} / \mathbb{Q}\right)$ is the internal direct product of $\Delta_{n}$ and $\Gamma_{n} . S$ will denote the set of places $\{\infty, \ell\}$ of $\mathbb{Q}$.

By virtue of the natural isomorphism $\Delta_{n} \rightarrow \Delta$, characters of $\Delta_{n}$ correspond to characters of $\Delta$. If $\delta \in \widehat{\Delta}$, we let $\delta_{n}$ denote the corresponding character in $\widehat{\Delta}_{n}$. Now, the idea is to view the group-ring $\mathbb{C}\left[G_{n}\right]$ as $\mathbb{C}\left[\Gamma_{n}\right]\left[\Delta_{n}\right]$. In doing this, we can define a projection $\pi_{n}(\delta): \mathbb{C}\left[G_{n}\right] \rightarrow \mathbb{C}\left[\Gamma_{n}\right]$ by extending $\delta_{n}$ linearly (over $\left.\mathbb{C}\left[\Gamma_{n}\right]\right)$.

Finally, fix an isomorphism $\nu: \mathbb{C}_{\ell} \rightarrow \mathbb{C}$ and let $\omega: \Delta \rightarrow \mathbb{C}^{\times}$be the composition of the Teichmüller character $\Delta \rightarrow \mathbb{C}_{\ell}^{\times}$with $\nu: \mathbb{C}_{\ell}^{\times} \rightarrow \mathbb{C}^{\times}$. Then given $\delta \in \widehat{\Delta}, \delta^{*}$ will denote $\omega \delta^{-1}$. Observe that since $\omega$ is odd, $\delta$ is even if and only if $\delta^{*}$ is odd.

Proposition 6.2 Let $\delta \in \widehat{\Delta}$. ( $\delta$ may be even or odd.)

$\operatorname{Fitt}_{\mathbb{Z}_{\ell} \llbracket \Gamma_{\infty} \rrbracket}\left(e_{\delta^{*}} \mathrm{Cl}_{\infty}\right)= \begin{cases}\lim _{\leftarrow n} \mathbb{Z}_{\ell} \pi_{n}\left(\delta^{*}\right)\left(\mathcal{J}\left(E_{n} / \mathbb{Q}, S\right)\right) & \text { if } \delta \neq 1 \\ \lim _{\leftarrow n} \mathbb{Z}_{\ell} \pi_{n}\left(\delta^{*}\right)\left(\left(1-(1+\ell) \sigma_{n}^{-1}\right) \mathcal{J}\left(E_{n} / \mathbb{Q}, S\right)\right) & \text { if } \delta=1\end{cases}$

where $\sigma_{n}=\left(1+\ell, E_{n} / \mathbb{Q}\right)$.

Proof. This stems from (3.1), which we reproduce for convenience:

$$
\mathcal{J}\left(E_{n} / \mathbb{Q}, S\right)=\frac{1}{2} e_{+} \operatorname{ann}_{\mathbb{Z}\left[G_{n}\right]}\left(\mathcal{O}_{E_{n}^{+}, S}^{\times} / \mathcal{E}_{n}^{+}\right) \oplus \mathbb{Z}\left[G_{n}\right] \theta_{E_{n} / \mathbb{Q}, S} .
$$

Let us deal with even characters $\delta \in \widehat{\Delta}$ first. For simplicity, we will assume that $\delta \neq 1$, though in fact the case $\delta=1$ is similar. (3.1) tells us that for each $n \geq 0, \mathbb{Z}_{\ell} \pi_{n}\left(\delta^{*}\right)\left(\mathcal{J}\left(E_{n} / \mathbb{Q}, S\right)\right)=\mathbb{Z}_{\ell}\left[\Gamma_{n}\right] \pi_{n}\left(\delta^{*}\right)\left(\theta_{E_{n} / \mathbb{Q}, S}\right)$. However, Iwasawa's construction of $\ell$-adic $L$-functions (see [19] and [40, Chapter 7]) shows that this lies in $\mathbb{Z}_{\ell}\left[\Gamma_{n}\right]$ and that the inverse limit of these ideals is generated by the algebraic $\ell$-adic $L$-function corresponding to the even character $\delta$. Mazur and Wiles' proof (see 24]) of the Main Conjecture of Iwasawa theory, and later Wiles' generalization of this (see [41]), show that this in turn is equal to the Fitting ideal appearing in the statement of the proposition. 
Now we turn to odd characters $\delta \in \widehat{\Delta}$. Referring to (3.1) again, we find that

$$
\mathbb{Z}_{\ell} \pi_{n}\left(\delta^{*}\right)\left(\mathcal{J}\left(E_{n} / \mathbb{Q}, S\right)\right)=\pi_{n}\left(\delta^{*}\right)\left(\operatorname{Fitt}_{\mathbb{Z}_{\ell}\left[G_{n}\right]}\left(\left(\mathcal{O}_{E_{n}^{+}, S}^{\times} / \mathcal{E}_{n}^{+}\right) \otimes_{\mathbb{Z}} \mathbb{Z}_{\ell}\right)\right) .
$$

This uses that $\left(\mathcal{O}_{E_{n}^{+}, S}^{\times} / \mathcal{E}_{n}^{+}\right) \otimes_{\mathbb{Z}} \mathbb{Z}_{\ell}$ is cocyclic as a $\mathbb{Z}_{\ell}\left[G_{n}\right]$-module so that, since $G_{n}$ is cyclic, the Fitting and annihilator ideals of $\left(\mathcal{O}_{E_{n}^{+}, S}^{\times} / \mathcal{E}_{n}^{+}\right) \otimes_{\mathbb{Z}} \mathbb{Z}_{\ell}$ agree. [13. Theorem 1] says in particular that this Fitting ideal is equal to that of $\mathrm{Cl}\left(E_{n}^{+}\right) \otimes_{\mathbb{Z}} \mathbb{Z}_{\ell}$. Combining the above and passing to limits completes the proof.

We observe the importance here of taking leading coefficients of $L$-functions at $s=0$ rather than just values. For $\delta$ even (i.e. $\delta^{*}$ odd), $\pi_{n}\left(\delta^{*}\right)\left(\mathcal{J}\left(E_{n} / \mathbb{Q}, S\right)\right.$ ) concerns $L$-functions which are non-zero at 0 , and we get the usual Stickelberger elements which are related to minus parts of class-groups via $\ell$-adic $L$-functions. However when $\delta$ is odd (i.e. $\delta^{*}$ is even), $\pi_{n}\left(\delta^{*}\right)\left(\mathcal{J}\left(E_{n} / \mathbb{Q}, S\right)\right.$ ) is concerned with $L$-functions having simple zeroes at 0 , which are related to plus parts of classgroups via cyclotomic units.

\section{$7 \quad \mathcal{J}_{E / F}^{r}$ and annihilation}

Let $\ell$ be an odd prime. Given $\alpha \in \mathcal{J}_{E / F}^{r}$ and $H \subseteq G=\operatorname{Gal}(E / F)$, choose any

$$
\beta \in \operatorname{ann}_{\mathbb{Z}_{\ell}\left[H^{\mathrm{ab}}\right]}\left(\operatorname{Tors} H_{\text {ét }}^{1}\left(\operatorname{Spec}\left(\mathcal{O}_{E^{[H, H]}, S}\right), \mathbb{Z}_{\ell}(1-r)\right)\right) .
$$

Then the $H$-component $B_{G}^{*}(\alpha)_{H}$ lies in $\mathbb{Q}_{\ell}\left[H^{\mathrm{ab}}\right]^{N_{G} H}$, the fixed points under the conjugation action by $N_{G} H$, the normalizer of $H$ in $G$. Assuming Conjecture 6.1(i), $B_{G}^{*}(\alpha)_{H} \cdot \beta \in \mathbb{Z}_{\ell}\left[H^{\mathrm{ab}}\right]^{N_{G} H}$. Choose $z_{H, \alpha, \beta} \in \mathbb{Z}_{\ell}[H]$ such that

$$
\pi\left(z_{H, \alpha, \beta}\right)=B_{G}^{*}(\alpha)_{H} \cdot \beta .
$$

Consider the composition

$$
\begin{gathered}
H_{\text {ét }}^{2}\left(\operatorname{Spec}\left(\mathcal{O}_{E, S(E)}\right), \mathbb{Z}_{\ell}(1-r)\right) \stackrel{\operatorname{Tr}_{E / E^{[H, H]}}}{\rightarrow} H_{\text {ét }}^{2}\left(\operatorname{Spec}\left(\mathcal{O}_{E^{[H, H]}, S}\right), \mathbb{Z}_{\ell}(1-r)\right) \\
B_{G}^{*} \stackrel{(\alpha)_{H} \cdot \beta}{\rightarrow} H_{\text {ét }}^{2}\left(\operatorname{Spec}\left(\mathcal{O}_{E^{[H, H]}, S}\right), \mathbb{Z}_{\ell}(1-r)\right) \\
\stackrel{j}{\rightarrow} H_{\text {ét }}^{2}\left(\operatorname{Spec}\left(\mathcal{O}_{E, S(E)}\right), \mathbb{Z}_{\ell}(1-r)\right)
\end{gathered}
$$

in which $j$ is induced by the inclusion of fields and $\operatorname{Tr}_{E / E^{[H, H]}}$ denotes the transfer homomorphism.

Assuming Conjecture 6.1(ii), this composition is zero. However, by Frobenius reciprocity for the cohomology transfer, for all $a \in H_{\text {êt }}^{2}\left(\operatorname{Spec}\left(\mathcal{O}_{E, S(E)}\right), \mathbb{Z}_{\ell}(1-\right.$ $r)$ )

$$
\begin{aligned}
0 & =j\left(\pi\left(z_{H, \alpha, \beta}\right) \operatorname{Tr}_{E / E^{[H, H]}}(a)\right) \\
& =j \cdot \operatorname{Tr}_{E / E^{[H, H]}}\left(z_{H, \alpha, \beta} \cdot a\right) \\
& =\left(\sum_{h \in \operatorname{Gal}\left(E / E^{[H, H]}\right)} h\right) z_{H, \alpha, \beta} \cdot a .
\end{aligned}
$$


Definition 7.1 In the situation of $\$ 6.1$ and $\$ 7$, let $I(E / F, r) \subseteq \mathbb{Z}_{\ell}[G]$ denote the left ideal generated by the elements $\left(\sum_{h \in \operatorname{Gal}\left(E / E^{[H, H]}\right)} h\right) z_{H, \alpha, \beta}$ as $\alpha, H$ and $\beta$ vary through all the possibilities above.

Theorem 7.2 If Conjecture 6.1 is true for all abelian intermediate extensions $E^{[H, H]} / E^{H}$ of $E / F$ then the left action of the left ideal $I(E / F, r)$ annihilates

$$
H_{\text {ét }}^{2}\left(\operatorname{Spec}\left(\mathcal{O}_{E, S(E)}\right), \mathbb{Z}_{\ell}(1-r)\right) .
$$

Remark. If $G$ is abelian in Definition 7.1 and Theorem 7.2, then

$$
I(E / F, r)=\mathcal{J}_{E / F}^{r} \cdot \operatorname{ann}_{\mathbb{Z}_{\ell}[G]}\left(\operatorname{Tors} H_{\text {êt }}^{1}\left(\operatorname{Spec}\left(\mathcal{O}_{E, S(E)}\right), \mathbb{Z}_{\ell}(1-r)\right)\right) .
$$

That is, $I(E / F, r)$ equals the left hand side of Conjecture 6.1(ii).

Proposition 7.3 In Definition 7.1, $I(E / F, r)$ is a two-sided ideal in $\mathbb{Z}_{\ell}[G]$.

Proof. In the notation of \$7, it suffices to show that

$$
w\left(\sum_{h \in \operatorname{Gal}\left(E / E^{[H, H]}\right)} h\right) z_{H, \alpha, \beta} w^{-1}
$$

lies in $I(E / F, r)$. Consider

$$
w\left(\sum_{h \in \operatorname{Gal}(E, E[H, H]} h\right) w^{-1}=\sum_{h \in \operatorname{Gal}\left(E / E^{\left[w H w^{-1}, w H w^{-1}\right]}\right)} h
$$

and $w z_{H, \alpha, \beta} w^{-1}$. Since $z_{H, \alpha, \beta}$ lies in $\mathbb{Z}_{\ell}[H]$ and maps to $B_{G}^{*}(\alpha) \beta$ in $\mathbb{Z}_{\ell}\left[H^{\mathrm{ab}}\right]$, we see that $w z_{H, \alpha, \beta} w^{-1}$ lies in $\mathbb{Z}_{\ell}\left[w H w^{-1}\right]$ and maps to $w B_{G}^{*}(\alpha)_{H} w^{-1} w \beta w^{-1}$ in $\mathbb{Z}_{\ell}\left[H^{\mathrm{ab}}\right]$. However, $w B_{G}^{*}(\alpha)_{H} w^{-1}=B_{G}^{*}(\alpha)_{w H w^{-1}}$ and $w \beta w^{-1}$ lies in

$$
\operatorname{ann}_{\mathbb{Z}_{\ell}\left[\left(w H w^{-1}\right)^{\mathrm{ab}]}\right]}\left(\operatorname{Tors} H_{\text {ét }}^{1}\left(\operatorname{Spec}\left(\mathcal{O}_{E\left[w H w^{-1}, w H w^{-1}\right], S}\right), \mathbb{Z}_{\ell}(1-r)\right)\right),
$$

completing the proof.

Proposition 7.4 Suppose that $F \subseteq K \subseteq E$ is a tower of number fields with $E / F$ and $K / F$ Galois. Then for $r=-1,-2,-3, \ldots$, the canonical homomorphism $\pi_{E / K}: \mathbb{Z}_{\ell}[\operatorname{Gal}(E / F)] \rightarrow \mathbb{Z}_{\ell}[\operatorname{Gal}(K / F)]$ satisfies

$$
\pi_{E / K}(I(E / F, r)) \subseteq I(K / F, r) .
$$

Proof. This follows easily from Lemma 4.1 and Proposition 5.3. 


\section{Relation to Iwasawa theory}

As discussed in the Introduction, the motivation for examining the behaviour of the fractional Galois ideal under changes of extension is to set up investigating a possible role in Iwasawa theory. Via the relationship of the fractional ideal with Stark-type elements (eg cyclotomic units in the case $r=0$ and Beilinson elements in the case $r<0$, discussed in 5 and 33 resp.), one might hope that an approach involving Euler systems would be fruitful here. A general connection of the fractional Galois ideal to Stark elements of arbitrary rank was demonstrated in [6], and the link of Stark elements with class-groups using the theory of Euler systems is discussed in [29, 25], so that a strategy as above would seem promising.

We conclude the paper with some speculation concerning what the noncommutative Iwasawa theory of Fukaya-Kato [15], Kato 20] and Ritter-Weiss 27. suggests about $\mathcal{J}_{E / F}^{r}$ of Definition 5.4 and $I(E / F, r)$ of Definition 7.1 .

It is worth pointing out, before we begin the recapitulation proper, that [15, 20, 27. often restrict to the situation where the extension fields are totally real, which tends to involve only one of the eigenspaces of complex conjugation acting on $\mathcal{J}_{E / F}^{r}$ and $I(E / F, r)$. We have tried to give some examples (for example, 86.2.1) which illustrate the expected role and properties of the other eigenspace.

Further, in this area there is an immense litany of conjectures (see [15, 7]) of which Stark's conjecture is approximately the weakest. All the constructions we have made are contingent only on the truth of Stark's conjecture, which is crucial for us but also seems fundamental; it is assumed, for example, in [28.

Following [20, let $\ell$ be an odd prime (denoted $p$ there), $F$ a totally real number field and $F_{\infty}$ a totally real Lie extension of $F$ containing $\mathbb{Q}\left(\zeta_{\ell^{\infty}}\right)^{+}$. Here, $\mathbb{Q}\left(\zeta_{\ell^{\infty}}\right)^{+}$is the union of the totally real fields $\mathbb{Q}\left(\zeta_{\ell^{n}}\right)^{+}=\mathbb{Q}\left(\zeta_{\ell^{n}}+\zeta_{\ell^{n}}^{-1}\right)$ over all $n \geq 1$. Let $G=\operatorname{Gal}\left(F_{\infty} / F\right)$, and assume that only finitely many primes of $F$ ramify in $F_{\infty}$. Fix a finite set $\Sigma$ of primes of $F$ containing the ones which ramify in $F_{\infty} / F$. Define $\Lambda(G)$ to be the Iwasawa algebra of $G$, given by $\Lambda(G)=\mathbb{Z}_{\ell} \llbracket G \rrbracket=\lim _{\leftarrow U} \mathbb{Z}_{\ell}[G / U]$, where the limit runs over all open normal subgroups of $G$.

Let $C$ denote the cochain complex of $\Lambda(G)$-modules given by

$$
\mathrm{RHom}\left(\mathrm{R} \Gamma_{\text {ét }}\left(\mathcal{O}_{\mathrm{F}_{\infty}}[1 / \Sigma], \mathbb{Q}_{\ell} / \mathbb{Z}_{\ell}\right), \mathbb{Q}_{\ell} / \mathbb{Z}_{\ell}\right),
$$

so that $H^{0}(C)=\mathbb{Z}_{\ell}$ with trivial $G$-action and $H^{-1}(C)=\operatorname{Gal}\left(M / F_{\infty}\right)$, the Galois group of the maximal pro- $\ell$ abelian extension of $F_{\infty}$ unramified outside $\Sigma$. The other $H^{i}(C)$ 's are zero and $\operatorname{Gal}\left(M / F_{\infty}\right)$ is a finitely generated torsion (left) $\Lambda(G)$-module. Let $F^{c y c} \subseteq F_{\infty}$ denote the cyclotomic $\mathbb{Z}_{\ell}$-extension and set $H=\operatorname{Gal}\left(F_{\infty} / F^{c y c}\right) \subseteq G$ so that $G / H \cong \mathbb{Z}_{\ell}$. As in [11, let

$$
S=\{f \in \Lambda(G) \mid \Lambda(G) / \Lambda(G) f \text { is finitely generated as a } \Lambda(H) \text {-module }\} .
$$

Then $S$ is an Ore set, which means that its elements may be inverted to form the localized ring $\Lambda(G)_{S}$, and there is an exact localization sequence of algebraic 
K-groups

$$
K_{1}(\Lambda(G)) \rightarrow K_{1}\left(\Lambda(G)_{S}\right) \stackrel{\partial}{\rightarrow} K_{0}\left(\Lambda(G), \Lambda(G)_{S}\right) \rightarrow K_{0}(\Lambda(G)) \rightarrow K_{0}\left(\Lambda(G)_{S}\right) .
$$

By [17, Iwasawa's conjecture concerning the vanishing of the $\mu$-invariant implies that the cohomology of the perfect complex $C$ vanishes when $S$-localized. This gives rise to a class $[C] \in K_{0}\left(\Lambda(G), \Lambda(G)_{S}\right)$. In the case of finite Galois extensions the class $[C]$ accounts for the Stickelberger phenomena (c.f. 34]) but on the other hand so do values of Artin $L$-functions. The main conjecture of non-commutative Iwasawa theory, described below following [20, makes this relation clear in terms of $\Lambda(G)_{S}$-modules.

There is an $\ell$-adic determinantal valuation which assigns to $f \in K_{1}\left(\Lambda(G)_{S}\right)$ and a continuous Artin representation $\rho$ a value $f(\rho) \in \overline{\mathbb{Q}}_{\ell} \cup\{\infty\}$. The main conjecture of non-commutative Iwasawa theory asserts that there exists $\xi \in$ $K_{1}\left(\Lambda(G)_{S}\right)$ such that (i) $\partial(\xi)=-[C]$ and (ii) $\xi\left(\rho \kappa^{r}\right)=L_{\Sigma}(1-r, \rho)$ for any even $r \geq 2$ where $\kappa$ is the $\ell$-adic cyclotomic character and $L_{\Sigma}(s, \rho)$ is the Artin $L$-function of $\rho$ with the Euler factors at $\Sigma$ removed.

The main conjecture of Iwasawa theory was formulated in 28 and studied in the series of papers 27] when the Lie group $G$ has rank zero or one. The case of $G=G L_{2}\left(\mathbb{Z}_{\ell}\right)$ is of particular interest in the study of elliptic curves $E / \mathbb{Q}$ without complex multiplication [11] and is proven for the $\ell$-adic Heisenberg group in [20]. For a comprehensive survey see [15.

Motivated by the main conjecture of Iwasawa theory, and more generally by the role of $\Lambda(G)$ in the arithmetic geometry of elliptic curves and their Selmer groups, there has been considerable ring-theoretic activity concerning $\Lambda(G)$ and $\Omega(G)=\Lambda(G) / \ell \Lambda(G)$ (see [1, 2, 3, 37, 38, 39]). The rings $\Lambda(G)$ and $\Omega(G)$ are examples of "just-infinite rings" which both satisfy the Auslander-Gorenstein condition and are thus amenable to Lie theoretic analysis.

In the survey article [1, a number of questions are posed. In particular the constructions of $\S 7$ are directly related to [1, Question G]: "Is there a mechanism for constructing ideals of Iwasawa algebras which involves neither central elements nor closed normal subgroups?"

Proposition 8.1 If $F_{\infty} / F$ is any $\ell$-adic Lie extension of a number field $F$ with Galois group $G$ then, under the assumption of $\$ 7$ for the finite intermediate subextensions $E / F$ for $r=-1,-2,-3, \ldots$ we may define a two-sided ideal

$$
I\left(F_{\infty} / F, r\right)=\lim _{\leftarrow E} I(E / F, r)
$$

in $\Lambda(G)$, where the limit is taken over finite Galois subextensions $E / F$ of $F_{\infty} / F$.

In view of the annihilation discussion of $\$ 7$ Proposition 8.1 suggests the following:

Question 8.2 What is the intersection of the canonical Ore set $S$ of [11, 20] with $I\left(F_{\infty} / F, r\right)$ ? 
In many ways the most interesting case is when $G=G L_{2}\left(\mathbb{Z}_{\ell}\right)(\ell \geq 7)$ arising from the tower of $\ell$-primary torsion points on an elliptic curve over $\mathbb{Q}$ without complex multiplication [10, 11. In this case one has particularly strong information concerning two-sided primes ideals of $\Lambda(G)$ - see 3 . There is a possibly alternative approach to the construction of fractional Galois ideals in $\mathbb{Q}_{\ell}[\operatorname{Gal}(K / \mathbb{Q})]$ based on assuming that a type of Stark conjecture holds for the Hasse-Weil $L$-function of the elliptic curve [35. It would be interesting to know whether this leads to the same two-sided ideal as Proposition 8.1.

\section{References}

[1] K. Ardakov and K. A. Brown. Ring-theoretic properties of Iwasawa algebras: a survey. Doc. Math., (Extra Vol.):7-33 (electronic), 2006.

[2] K. Ardakov and K. A. Brown. Primeness, semiprimeness and localisation in Iwasawa algebras. Trans. Amer. Math. Soc., 359(4):1499-1515 (electronic), 2007.

[3] K. Ardakov, F. Wei, and J. J. Zhang. Reflexive ideals in Iwasawa algebras. To appear in Advances in Mathematics.

[4] Armand Brumer. On the units of algebraic number fields. Mathematika, $14: 121-124,1967$.

[5] Paul Buckingham. The canonical fractional Galois ideal at $s=0$. To appear in the Journal of Number Theory.

[6] Paul Buckingham. PhD thesis, University of Sheffield. 2008.

[7] D. Burns. Leading terms and values of equivariant motivic $L$-functions. To appear in the special volume of the Quarterly Journal of Pure and Applied Mathematics on the occasion of Tate's 80th birthday.

[8] Pierrette Cassou-Noguès. Valeurs aux entiers négatifs des fonctions zêta et fonctions zêta p-adiques. Invent. Math., 51(1):29-59, 1979.

[9] J. Coates and W. Sinnott. On $p$-adic $L$-functions over real quadratic fields. Invent. Math., 25:253-279, 1974.

[10] John Coates. Fragments of the $\mathrm{GL}_{2}$ Iwasawa theory of elliptic curves without complex multiplication. In Arithmetic theory of elliptic curves (Cetraro, 1997), volume 1716 of Lecture Notes in Math., pages 1-50. Springer, Berlin, 1999.

[11] John Coates, Takako Fukaya, Kazuya Kato, Ramdorai Sujatha, and Otmar Venjakob. The $\mathrm{GL}_{2}$ main conjecture for elliptic curves without complex multiplication. Publ. Math. Inst. Hautes Études Sci., (101):163-208, 2005. 
[12] John Coates and Warren Sinnott. An analogue of Stickelberger's theorem for the higher K-groups. Invent. Math., 24:149-161, 1974.

[13] Pietro Cornacchia and Cornelius Greither. Fitting ideals of class groups of real fields with prime power conductor. J. Number Theory, 73(2):459-471, 1998.

[14] Pierre Deligne and Kenneth A. Ribet. Values of abelian $L$-functions at negative integers over totally real fields. Invent. Math., 59(3):227-286, 1980 .

[15] Takako Fukaya and Kazuya Kato. A formulation of conjectures on $p$-adic zeta functions in noncommutative Iwasawa theory. In Proceedings of the St. Petersburg Mathematical Society. Vol. XII, volume 219 of Amer. Math. Soc. Transl. Ser. 2, pages 1-85, Providence, RI, 2006. Amer. Math. Soc.

[16] B. H. Gross. On the values of Artin $L$-functions. Unpublished preprint.

[17] Yoshitaka Hachimori and Romyar T. Sharifi. On the failure of pseudonullity of Iwasawa modules. J. Algebraic Geom., 14(3):567-591, 2005.

[18] David R. Hayes. Stickelberger functions for non-abelian Galois extensions of global fields. In Stark's conjectures: recent work and new directions, volume 358 of Contemp. Math., pages 193-206. Amer. Math. Soc., Providence, RI, 2004 .

[19] Kenkichi Iwasawa. Lectures on p-adic L-functions. Princeton University Press, Princeton, N.J., 1972. Annals of Mathematics Studies, No. 74.

[20] Kazuya Kato. Iwasawa theory of totally real fields for Galois extensions of Heisenberg type. Preprint.

[21] Tomio Kubota and Heinrich-Wolfgang Leopoldt. Eine $p$-adische Theorie der Zetawerte. I. Einführung der $p$-adischen Dirichletschen $L$-Funktionen. J. Reine Angew. Math., 214/215:328-339, 1964.

[22] Serge Lang. Algebra. Addison-Wesley Publishing Company Advanced Book Program, Reading, MA, second edition, 1984.

[23] J. Martinet. Character theory and Artin $L$-functions. In Algebraic number fields: L-functions and Galois properties (Proc. Sympos., Univ. Durham, Durham, 1975), pages 1-87. Academic Press, London, 1977.

[24] B. Mazur and A. Wiles. Class fields of abelian extensions of Q. Invent. Math., 76(2):179-330, 1984.

[25] Cristian D. Popescu. Rubin's integral refinement of the abelian Stark conjecture. In Stark's conjectures: recent work and new directions, volume 358 of Contemp. Math., pages 1-35. Amer. Math. Soc., Providence, RI, 2004. 
[26] Daniel Quillen. Higher algebraic $K$-theory. I. In Algebraic $K$-theory, I: Higher K-theories (Proc. Conf., Battelle Memorial Inst., Seattle, Wash., 1972), pages 85-147. Lecture Notes in Math., Vol. 341. Springer, Berlin, 1973.

[27] Jürgen Ritter and Alfred Weiss. Toward equivariant Iwasawa theory. Part I, Manuscripta Math., 109(2):131-146, 2002; Part II, Indag. Math. (N.S.), 15(4):549-572, 2004; Part III, Math. Ann., 336(1):27-49, 2006; Part IV, Homology, Homotopy Appl., 7(3):155-171, 2005 (electronic).

[28] Jürgen Ritter and Alfred Weiss. The lifted root number conjecture and Iwasawa theory. Mem. Amer. Math. Soc., 157(748):viii+90, 2002.

[29] Karl Rubin. Stark units and Kolyvagin's "Euler systems". J. Reine Angew. Math., 425:141-154, 1992.

[30] Jean-Pierre Serre. Linear representations of finite groups. Springer-Verlag, New York, 1977. Translated from the second French edition by Leonard L. Scott, Graduate Texts in Mathematics, Vol. 42.

[31] Victor Snaith. Equivariant motivic phenomena. In Axiomatic, enriched and motivic homotopy theory, volume 131 of NATO Sci. Ser. II Math. Phys. Chem., pages 335-383. Kluwer Acad. Publ., Dordrecht, 2004.

[32] Victor P. Snaith. Explicit Brauer induction, volume 40 of Cambridge Studies in Advanced Mathematics. Cambridge University Press, Cambridge, 1994. With applications to algebra and number theory.

[33] Victor P. Snaith. Relative $K_{0}$, annihilators, Fitting ideals and Stickelberger phenomena. Proc. London Math. Soc. (3), 90(3):545-590, 2005.

[34] Victor P. Snaith. Stark's conjecture and new Stickelberger phenomena. Canad. J. Math., 58(2):419-448, 2006.

[35] Jeffrey Stopple. Stark conjectures for CM elliptic curves over number fields. J. Number Theory, 103(2):163-196, 2003.

[36] John Tate. Les conjectures de Stark sur les fonctions $L$ d'Artin en $s=0$, volume 47 of Progress in Mathematics. Birkhäuser Boston Inc., Boston, MA, 1984. Lecture notes edited by Dominique Bernardi and Norbert Schappacher.

[37] Otmar Venjakob. On the structure theory of the Iwasawa algebra of a p-adic Lie group. J. Eur. Math. Soc. (JEMS), 4(3):271-311, 2002.

[38] Otmar Venjakob. A non-commutative Weierstrass preparation theorem and applications to Iwasawa theory. J. Reine Angew. Math., 559:153-191, 2003. With an appendix by Denis Vogel.

[39] Otmar Venjakob. On the Iwasawa theory of $p$-adic Lie extensions. Compositio Math., 138(1):1-54, 2003. 
[40] Lawrence C. Washington. Introduction to cyclotomic fields, volume 83 of Graduate Texts in Mathematics. Springer-Verlag, New York, second edition, 1997.

[41] A. Wiles. The Iwasawa conjecture for totally real fields. Ann. of Math. (2), 131(3):493-540, 1990. 